\title{
Conformal mapping and efficient boundary element method without boundary elements for fast vortex particle simulations
}

\author{
P. Deglaire*, O. Ågren, H. Bernhoff, M. Leijon \\ The Ångström Laboratory, Swedish Centre for renewable Energy conversion, Division for Electricity and Lightning Research, \\ Department of Engineering Sciences, Uppsala University, Box 534, SE-751 21 Uppsala, Sweden
}

Received 22 September 2006; received in revised form 2 February 2007; accepted 27 March 2007

Available online 12 April 2007

\begin{abstract}
In this paper, a revitalization of conformal mapping methods applied to fluid flows in two dimensions is proposed. The present work addresses several important issues concerning their application for vortex particle flow solvers. Difficulties of past conformal based method are reviewed. One difficulty concerns the ability of a mapping procedure to represent complicated shapes. The present paper improves past algorithms to be able to map new shapes, including multiply connected domains. A new fast procedure allows transferring a set of points in the mapped simplified plane to the complicated domain and vice versa. After a mapping construction, it is demonstrated how basic exact solutions to potential flow problems with vortices can be put in a new form which provides a faster and more accurate computation than with distributed singularity methods.
\end{abstract}

(c) 2007 Elsevier Masson SAS. All rights reserved.

Keywords: Conformal mapping; Laurent's series expansion; Fast imaging

\section{Introduction: Conformal mapping and aerodynamics}

A conformal mapping is a transformation from the complex plane into the complex plane, which preserves the local angles. Riemann [1] showed that there exists one unique conformal map, which transforms the exterior of any shape (here a single airfoil) into the exterior of the unit disk. In the case of $N_{w}$ wing sections, the existence of the mapping transformation is given by the Koebe theorem [2]: Every $n$-ply connected region can be conformally mapped onto an exterior region which is bounded by $n$ circles.

In the past, such transformations have been extensively used to provide exact solutions of Laplace's equation with Dirichlet type boundary conditions.

In aerodynamics, Laplace equation arises when the flow is assumed irrotational and incompressible. From the incompressibility assumption, the streamfunction is defined and Laplace equation is obeyed with Dirichlet boundary conditions. Although ideal flows have limited practical interest, the Laplace equation and more generally the constant curl Laplacian equation [3] also arises in problems involving multiple bodies in motion with vortices. The present

\footnotetext{
* Corresponding author. Tel.: +461847135 66; fax: +46184713000.

E-mail address: paul.deglaire@ angstrom.uu.se (P. Deglaire).
} 
paper addresses a fast way of solving this situation and particularly the aspect of a fast and accurate evaluation of the body influence on the vortices in the context of vortex particle methods.

Conformal mapping for one single wing, introduced by Joukowsky, has been used since the early days of aerodynamics. The first full flexible mapping, due to Theodorsen and Garrick [4], can be applied to a wide variety of wing sections. However, their mapping presents convergence problems for wing profiles which are not smooth enough. In 1980 Ives [5] introduces a fast method by adding fast Fourier transforms (FFT) to the previous work of Theodorsen. With this improvement, the mapping of a single wing is efficiently computed (it takes a few milliseconds on a modern PC). The wing pressure distribution can then be deduced from the standard analytical solution for inviscid irrotational flow around a circle, see Section 8.

The more widespread panel method was introduced a few years before the implementation of FFT's to conformal mapping. In panel methods, the geometry is solved with a set of potential singularities directly on the wing profile in the physical plane. In today's aerodynamics, the mapping of one wing to a circle is still used in the elegant multipoint inverse design procedure originally due to Eppler [6,7].

Concerning multielement airfoil analysis via conformal mapping, blades in cascade and biplane were mapped first by Garrick [8] and the modern methods were developed in the 1980's by Halsey [9], Ives [5] and Wegmann [10]. Halsey's method can be generalized to four or even $N$-body mapping, while Ives method seems to be restricted to two bodies.

In the general study of aerodynamic flows, mapping methods are restricted to two dimensions.

The main identified drawback of conformal mapping analysis for two dimensional flows is the split of the direct problem is split into two. First, the geometry has to be transformed into a simpler one. Then the problem has to be solved in the simple geometry and the solution retransferred to the original geometry. Therefore the sum of the computation time of the transfers and of the solution of the modified problem has to be smaller than the direct solution by other methods.

To be more specific, the mapping methods are less useful if the transfer requires a long set of intricate functions to be evaluated iteratively and the simplified problem necessitates a long computation time, although it might provide an exact solution. These are the two goals of the present paper: to show that even for complicated situations the transfer can be made efficiently and secondly, to show that the simplified problem can be solved efficiently. The advantage of the method with respect to vortex particle applications will be emphasized in Section 7.

\section{Vortex particle method}

The present paper exploits the developments made in vortex particle methods during the last two decades and intends to show how a conformal mapping scheme could be an efficient alternative in two dimensions to the panel method. The vortex particle method has proved to be very accurate for unsteady flows around: bluff bodies [11], circles, airfoils at high angles of attack [12], or undergoing dynamic stall [13].

Throughout the following, all flows are assumed incompressible. Vortex particle methods are based on the vorticity equation obtained by taking the curl of the Navier-Stokes equation. In two dimensions, the vorticity equation is:

$$
\frac{\partial \underline{\omega}}{\partial t}+(\underline{u} \cdot \nabla) \underline{\omega}=v \nabla^{2} \underline{\omega}
$$

where $\underline{\omega}=\nabla \times \underline{u}=\omega e_{z}$ is the vorticity in two dimensions, and $v$ is the kinematic viscosity of the fluid considered.

Usually Eq. (1) is split into a convection and diffusion step.

First a convection step is considered:

$$
\frac{\mathrm{d} \omega^{(n)}}{\mathrm{d} t}=0 .
$$

Then, to include diffusion the next equation to consider at the same time step is

$$
\frac{\partial \omega^{(n+1)}}{\partial t}=v \nabla^{2} \omega^{(n)} \text {. }
$$

The idea of the particle method is to discretize the vorticity $\omega$ onto a set of vortex particles with a given vorticity kernel, which, far from the rotational area or core, presents a logarithmic potential. Far from all cores, the equations are that of an ideal fluid. Each particle convects with the fluid velocity induced by all other causes according to Eq. (2). 
In this respect the method is essentially mesh free and only focuses on the region of interest. The diffusion in Eq. (3) can be addressed by several standard means with the random walk method [14] or the more sophisticated particle strength exchange method [15] redistributing the vorticity of each particle to its neighbors.

To fulfill Eq. (2), the action of all external causes on each particle has to be summed. This action comes from three main groups: first the action of all the other particles in the fluid, second the action of the boundaries and third the action of the asymptotic flow.

Two decades ago, to compute the action of all other particles was too computationally demanding: to convect the particles in time, the velocity induced by the $N_{v}$ vortex particles has to be computed for the $N_{v}$ particles resulting in $\mathrm{O}\left(N_{v}^{2}\right)$ operations. Typically a large number of particles ranging from 10000 to 100000 have to be considered for accurate simulations. This implies computationally expensive methods. However the discovery of Fast Multipole Methods (FMM) by Greengard and Rokhlin [16] resulted in an $\mathrm{O}\left(N_{v} \log N_{v}\right)$ operations, or even as low as $\mathrm{O}\left(N_{v}\right)$ operations making the method very competitive.

When a Fast Multipole method is used, the action of the boundary should remain a small fraction of the total computation time which scales as $\mathrm{O}\left(N_{v}\right)$.

The action of the vortices on the boundaries and then the action induced by bodies on particles are usually examined by the inviscid potential flow panel method developed by Hess and Smith [17].

The basic implementation assumes that the body can be represented by a continuous line of vorticity which is split into a number of elements $N_{\text {Panel }}$ called panels. In the case of flat panels (with linear vorticity variation), this leads to solve a linear problem $A \Gamma=B$ where $A$ is dense. The right-hand side $B$ has $N_{\text {Panel }}$ lines and is formed by the computation of the velocity induced by all other causes other than the body on some control points. These are usually defined in the middle of each panel. In the context of particle simulations for non-deformable structures, the matrix $A$ is inversed and stored at the beginning of the simulation. The unknown body vorticity $\Gamma$ is found from $B$ by a simple matrix multiplication at each time step.

This method has shown to give fast and accurate results for steady inviscid flows around both single and multiple bodies without vortices.

The crucial difference between steady and unsteady flow with vortices is that the velocity needs to be evaluated only at the boundaries in the steady case and can be found directly from the vorticity values at the nodes. With vortices included, the action of the boundary is evaluated by summing all contributions from each panel. This requires a long computation time if a large number of panels and particles are present (see Table 1).

Moreover, the method relies heavily on a good generation of the vorticity from the bodies. In particular one approach is to diffuse the vorticity into the flow by releasing particles at a very small distance from the body surface. The panel methods encounters problem as it solves the potential flow no through boundary problem only in a fix number of pre-defined control points: when the vortex particles are too close to the body, unrealistic results [18] are obtained: substantial and non-physical edge effects [19] are present at the end of each panel for the most often used method, which assumes linear geometric element with linear variation of vorticity.

These aspects have been pointed out in a recent paper by Ramachandran [19] and solved using cubic geometry panel methods. If flat panel have to be used for particle methods and high Reynolds number flows are considered, the number of panels should be increased to ensure that the vortices very close to the body are subjected to a smooth contribution from the body. This results in a substantial increase in the computation time (see Table 1).

The conformal mapping transformation does not have such deficiencies since the no through boundary condition is solved exactly on the whole boundary. The method of images [20] is valid even if the vortex particles are very close to the body. Section 4 and the fast imaging procedure shows how to treat images efficiently. The use of conformal mapping does not add any extra assumption on the slip velocity on the body surface contrary to representation by piecewise linear, parabolic or cubic vorticity sheet. It can be considered as exact as no points are privileged in the body surface and even mesh free. In Section 7, the computational efficiency of the conformal mapping developed here is compared against the linear panel method.

On the other hand, panel methods are able to treat directly arbitrary geometry. If a mapping method would be superior, the mapping method also has to treat arbitrary geometries. The transfer between mapped and real plane has to be made without adding a significant computation time. This issue is addressed in Section 3. 


\section{Fast transfer and Laurent's series}

It is important to adapt the mapping algorithm to ensure a good transfer from the mapped plane onto the real plane and vice versa.

\subsection{From the circles to the airfoils}

The conformal mapping transformation is analytic. The transform has therefore a Laurent series expansion. One of the goals of the present paper is to describe a numerical method to find the transform of one circle into a given wing profile and more generally, $N_{w}$ circles into $N_{w}$ given wing profiles. The advantages of using Laurent's series representation will be stressed.

For a single wing, the airfoil coordinates are given by a set of discrete coordinates $\left(x_{i}, y_{i}\right)$ converted into a set of complex number $z_{i}$. A conformal transformation $f$ in the form of Laurent' series is:

$$
z=f(s)=s+\sigma_{0}+\sum_{k=1}^{N} \frac{c_{k} b^{k}}{s^{k}},
$$

where $\sigma_{0}$ and $c_{k}$ are complex numbers and $N$ is the number of terms retained. The function $f$ transforms a circle $s_{c}$ of radius $b$ in the $s$-plane into the wing section $z_{c}$ in the $z$-plane, represented by a set of discrete points.

$$
\begin{aligned}
& s_{c}=b \mathrm{e}^{\mathrm{i}\left(\eta+\eta_{\mathrm{TE}}\right)}, \\
& z_{c}=f\left(s_{c}\right) .
\end{aligned}
$$

The angle $\eta_{\mathrm{TE}}$ represents the airfoil trailing edge angle in the $s$-plane. The series in Eq. (4) is convergent on and outside the circle boundary $s_{c}$. The aim is to find the optimal $\sigma_{0}$ and $\left\{c_{k}\right\}_{k \in \mathbb{N}}$, which approaches as close as possible the shape given by interpolation of the points $z_{i}$ of the airfoil section. This method will be described in Section 4.7.

For the multicomponent section, the transform from $N_{w}$ circles into $N_{w}$ given wing profiles will be derived as:

$$
z=g(s)=s+\sum_{n=1}^{N_{w}} \sum_{k=1}^{N} \frac{c_{n, k} b_{n}^{k}}{\left(s-s_{\text {center }, n}\right)^{k}},
$$

where $N_{w}$ is the number of wings, and $\left\{c_{n, k}\right\}_{k \in \mathbb{N}, n \in \mathbb{N}}$ are complex numbers. The function $g$ transforms $N_{w}$ circles $s_{c, n}$ in the $s$-plane into $N_{w}$ wing sections $z_{c, n}$ in the $z$-plane, represented by a given set of discrete points.

$$
\begin{aligned}
& s_{c, n}=s_{\text {center }, n}+b_{n} \mathrm{e}^{\mathrm{i}\left(\eta+\eta_{\mathrm{TE}, n}\right)}, \\
& z_{c, n}=g\left(s_{c, n}\right),
\end{aligned}
$$

where $b_{n}$ is the radius of the $n$th circle in the $s$-plane, $s_{\mathrm{center}, n}$ its center and $\eta_{\mathrm{TE}, n}$ is the angle representing the trailing edge.

In particular for a periodic problem, assuming that the $n$th blade is obtained from the first one by atation of $n \Delta \beta$, the $g$ transform can be simplified into

$$
z=g(s)=s+\sum_{n=1}^{N_{w}} \sum_{k=1}^{N} \exp [\mathrm{i}(n-1)(k+1) \Delta \beta] \frac{c_{1, k} b_{1}^{k}}{\left(s-s_{\text {center }, 1} \exp (\mathrm{i}(n-1) \Delta \beta)\right)^{k}}
$$

and the $N_{w}$ circles representing the $N_{w}$ wings will be defined as

$$
\begin{aligned}
& s_{c, n}=s_{\text {center }, 1} \exp [\mathrm{i}(n-1) \Delta \beta]+b_{1} \mathrm{e}^{\mathrm{i}\left[\eta+\eta_{\mathrm{TE}, 1}+(n-1) \Delta \beta\right]}, \\
& z_{c, n}=g\left(s_{c, n}\right) .
\end{aligned}
$$

In other words, when the system is periodic, only the coefficients for the first wing need to be established to produce the full section. 


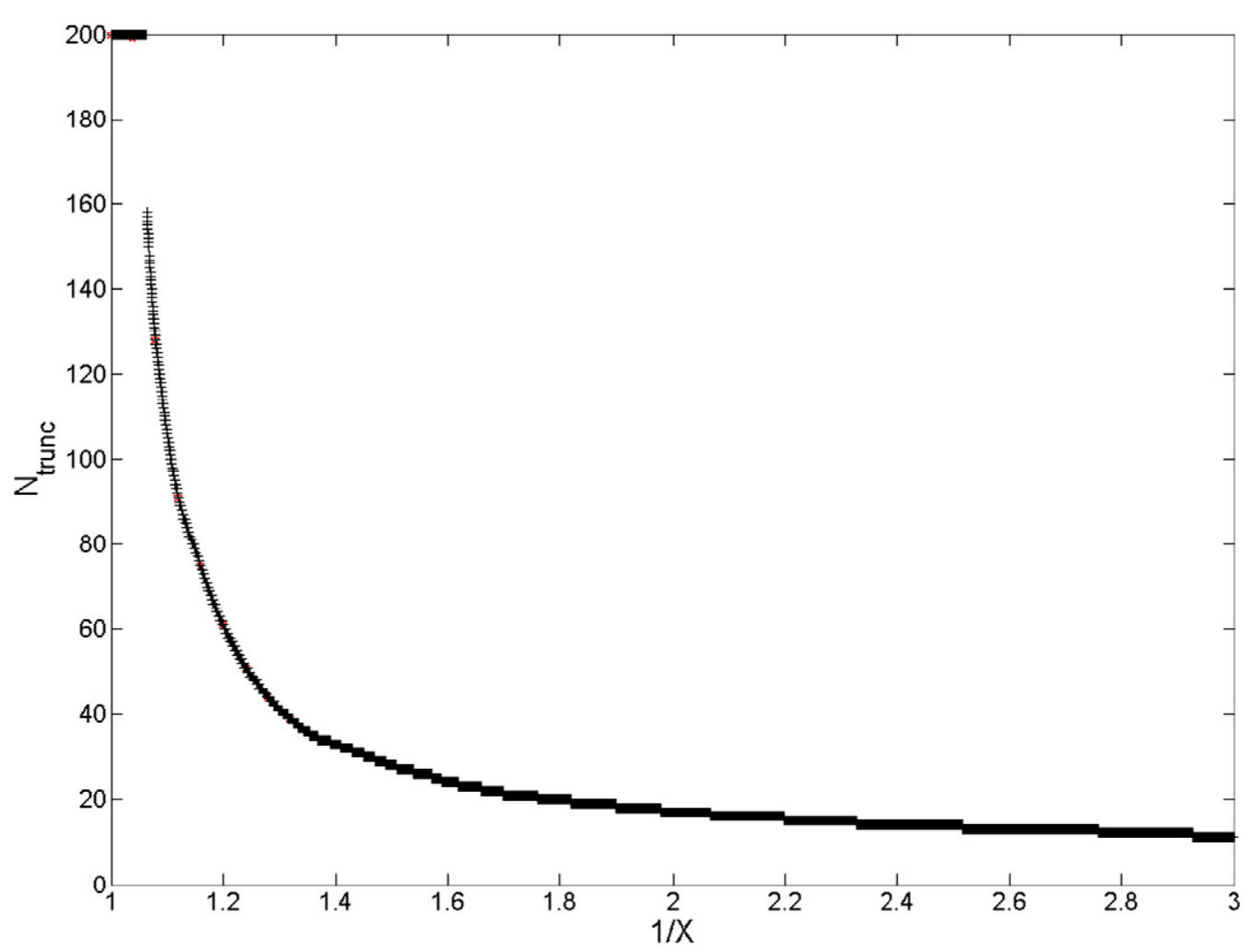

Fig. 1. Number of terms needed for the evaluation of the mapping function, $N_{\text {trunc }}$ as a function of the non-dimensional distance form the origin $1 / X$ in the $s$-plane. The wanted accuracy is set to $\varepsilon_{\text {want }}=10^{-10}$. The initial number of coefficients in the mapping function is $N=200$.

The mapping of circles onto given complicated shapes has been implemented in the same form of Laurent series as in the work of Prosnak [21]. However, this form is ideal for the fast computation of the mapping function in a significant number of points outside the circle (i.e. in the vortex particle positions) what was not stressed in Prosnak [22].

We will emphasize this by an illustrating example. We consider a single wing, and assume that the $N=200$ mapping coefficients are determined. We now place a set of 10000 points $s$ uniformly distributed inside the annulus of inner radius $b$ and outer radius $3 b$. The inner radius represents the wing in the mapped plane. We would like to evaluate as quickly as possible the position $z$ of these points in the real, airfoil plane. After some basic algebra, we find that it is enough to use only the first $N_{\text {trunc }}$ terms in the series for a wanted precision $\varepsilon_{\text {want }}$ where $N_{\text {trunc }}$ is given by:

$$
N_{\text {trunc }} \geqslant \frac{\ln \left|\varepsilon_{\text {want }}\right|-\ln A_{N_{\text {trunc }}}-\ln \left(1-X^{N}\right)+\ln (1-X)}{\ln X}-1
$$

with $A_{N_{\text {trunc }}}=\max _{k \in\left[N_{\text {trunc }}, N\right]}\left|c_{k}\right|$ and $X=|b / s|$ is the inverse radius of the non-dimensional position of the point in the circle plane.

This formula is valid only for points outside of the circle such that $X<1$.

If the wanted accuracy is equal to $10^{-10}$, the number of terms to be used in the evaluation is plotted in Fig. 1. We notice that the full number of coefficients which needs to be used are $N$ only at the boundary and close to it. As soon as the points are away from the circle by half a radius, the number of necessary terms is already less than 30 . A simple truncation criteria is then implemented and provides a fast evaluation of the Laurent's series.

The usefulness of this method is important in the case of multiply connected domain: from $N N_{w}$ terms in the series to be evaluated, it is possible to show that the maximum amount of needed terms is of the order of $N$ to the leading order. In other words, the evaluation of the mapping function in the $N_{w}$ case does not require more computation time than in the simply connected case.

From a practical point of view the Laurent's series are evaluated in a negligible computational time: in the order of ms on a normal PC and for 10000 points both for the simply and multiply connected case. 


\subsection{From the airfoils back to the circles}

Concerning particle methods, it has been found to be very practical to also look for the inverse of transformation $f$ in Eq. (4). The idea will be helpful in Section 4 considering the iterative multicomponent mapping.

The procedure follows: take a circle $C^{\prime}$ with center $z_{c}^{\prime}$ in the $z$ plane with a radius $b^{\prime}$ which includes the image of the $s$-plane circle by $f$. Put $2 P$ points uniformly on this circle. Then generate a polar grid in the $s$-plane which corresponds to an annulus bounded by the circle representing the airfoil in the $s$-plane and which image will completely contain the circle $C^{\prime}$. Compute the value of $f$ at all grid points. Extrapolate from the grid points values, the position of the points which are mapped onto the $2 P$ points in the circle $z_{c}^{\prime}$. Make an FFT using these positions. Using the coefficients $\left\{d_{k}\right\}_{k \in \mathbb{N}, n \in \mathbb{N}}$ found, the inverse transform can be written for every point outside and on the circle $C^{\prime}$ as

$$
s=f^{\prime}(z)=z+d_{0}+\sum_{k=1}^{N} \frac{d_{k} b^{\prime}}{z^{k}} .
$$

If points between the airfoil and the circle $C^{\prime}$ have to be found, the grid is directly used to find their positions in the $s$-plane.

If applied directly in the airfoil plane, the area between the airfoil and the smallest circle which encloses the airfoil can be large. Therefore the number of grid points can be very important. To alleviate this problem, we will use this procedure after one inverse Karman Trefftz mapping (see Section 4.7).

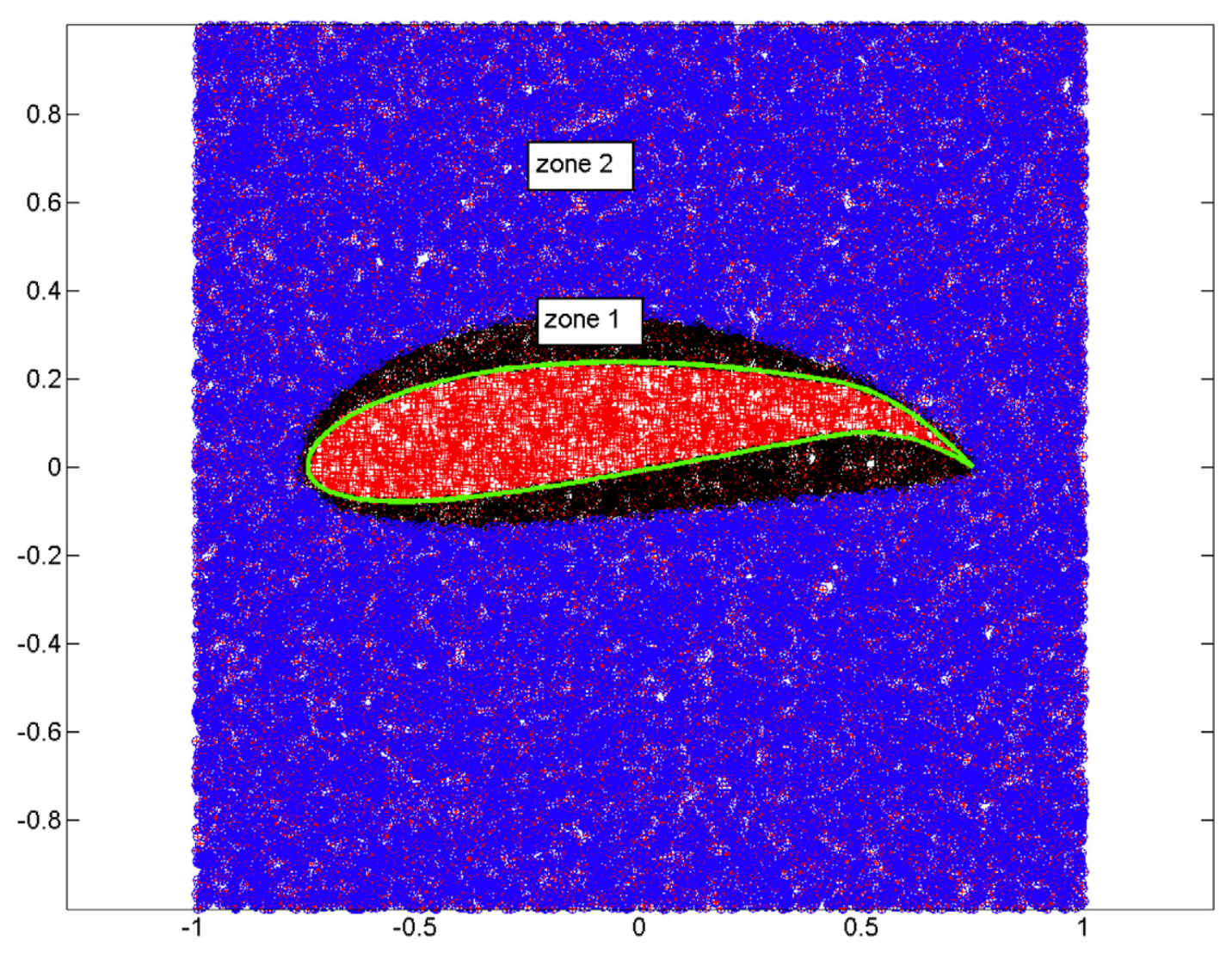

Fig. 2. Plot of the two zones considered in the inverse mapping of a NACA8818 airfoil, 50000 particles are uniformly distributed in a square box. Zone 1 will make use of the grid interpolation whereas zone 2 will use the Laurent's series to get the inverse images. 


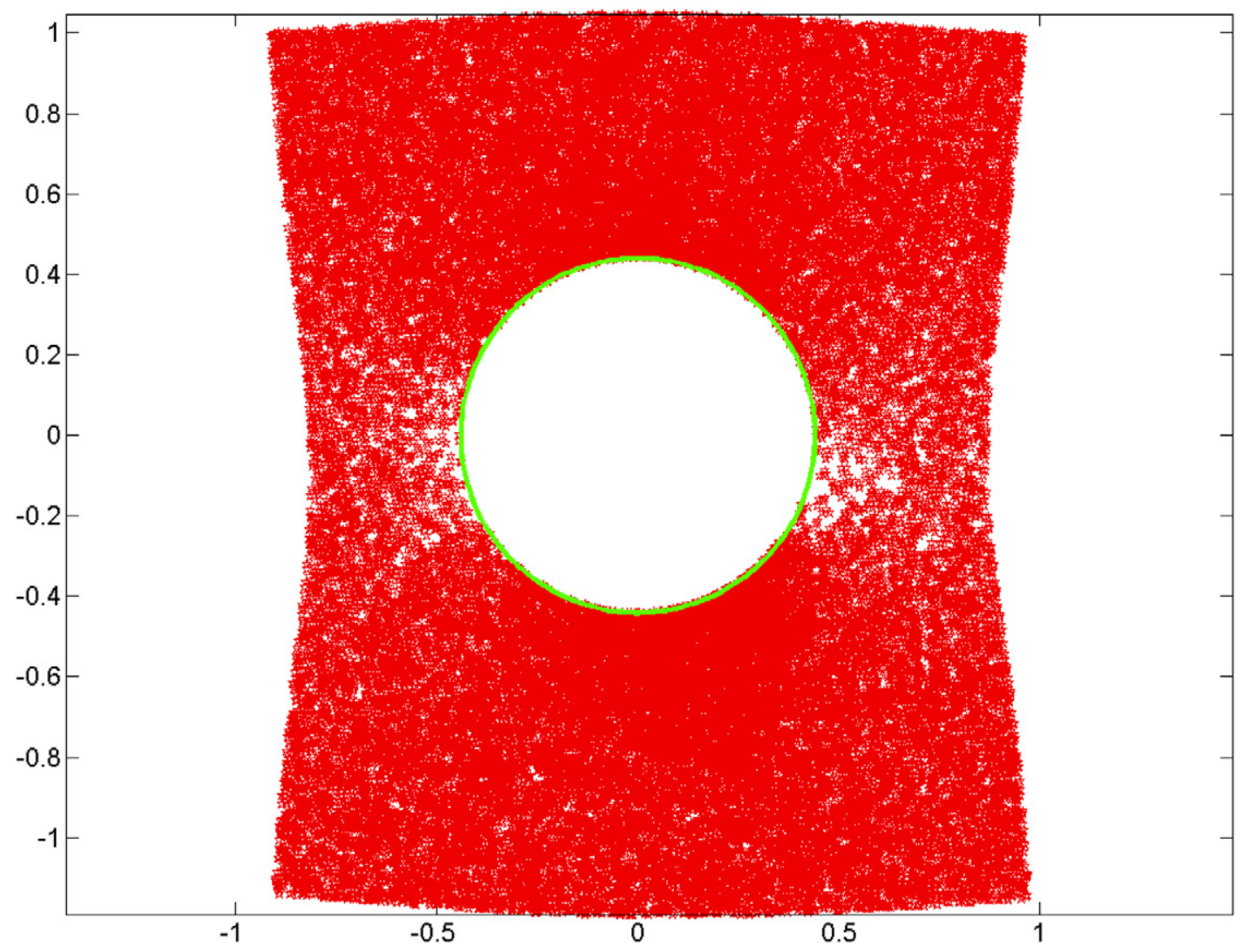

Fig. 3. Plot of the inverse images in the $s$-plane through the inverse mapping of a NACA8818 airfoil of the particles considered in Fig. 3. A uniform distribution of points in the $z$ plane (Fig. 3) does not correspond to a uniform distribution of points around the circle. The points which are found inside the circle in the $s$-plane are removed.

The same procedure applies to the multiply connected case. The inverse of the mapping function can be put in the following form outside of each circle $C_{w}^{\prime}$.

$$
s=g^{\prime}(z)=z+\sum_{n=1}^{N_{w}} \sum_{k=1}^{N} \frac{d_{n, k} b_{n}^{\prime}{ }^{k}}{\left(z-z_{\text {center }, n}^{\prime}\right)^{k}} .
$$

It is nearly as fast to go from one plane to the other and vice versa for both the simply connected and multiply connected case.

As an example, 50000 particles are uniformly distributed in a box around a NACA8818 airfoil. In Fig. 2, zone 1 corresponds to particles obtained via the grid interpolation, zone 2 using Laurent's series. A uniform distribution of points in the airfoil plane does not result in a uniform set of points in the circle plane see Fig. 3. This result emphasizes the importance of the fast inverse procedure with regards to random walk simulations for instance.

The absence of a general and fast inverse mapping procedure has limited the application of conformal mapping methods in vortex particles simulations to shapes where both the mapping and its inverse are known (Joukowsky airfoils [23] and Karman Trefftz profiles).

\section{Fast solutions in the simplified plane}

\subsection{General statements}

From a two-dimensional complicated turbine or other multicomponent geometries, conformal maps convert them into a new simple geometry, where an analytical or easy-to-obtain solution exists. In particular for the vortex particle 
method, the no penetration condition on the body is first to be ensured. The inviscid irrotational flow problem is solved for an arbitrary contour or $N_{w}$ contours. A complex potential $F$ exists under these assumptions, and is given by

$$
F=F(s)=\varphi+\mathrm{i} \psi,
$$

where $s$ is a complex number. The potential $\varphi$ and the streamfunction $\psi$ satisfy Cauchy-Riemann equations.

The velocity $V=u+\mathrm{i} v$ in the $s$-plane is deduced from $F$ as

$$
V=\frac{\overline{\mathrm{d} F}}{\mathrm{~d} s}
$$

where the bar stands for complex conjugate throughout this manuscript.

For a conformal mapping from the $s$-plane to the $z$ plane,

$$
z=f(s),
$$

the velocity in the $z$ plane is given (in every points where $f$ and $F$ are analytic) by

$$
V=\frac{\overline{\mathrm{d} F}}{\mathrm{~d} s} \frac{1}{f^{\prime}(s)} .
$$

The number of operations to find the velocity in $N_{V}$ points is very limited due to the adaptation of Section 3 to the derivative of the mapping function.

Basic solutions for one and $N_{w}$ circle flows will now be reviewed.

\subsection{Single wing and vortices: the fast imaging procedure}

One of the most well-known solutions stands for a circle of radius $b$ placed in a flow containing $N_{V}$ vortices. Following Milne-Thomson [20], the complex potential of the flow to be evaluated in the point $s$ is given by:

$$
F(s)=V_{0} \mathrm{e}^{-\mathrm{i} \alpha} s+V_{0} \mathrm{e}^{\mathrm{i} \alpha} \frac{b^{2}}{s}+\frac{\mathrm{i} \Gamma_{C}}{2 \pi} \log (s)+\sum_{v=1}^{N_{v}} \frac{\mathrm{i} G_{v}}{2 \pi}\left[\log \left(s-s_{V}\right)-\log \left(s-\frac{b^{2}}{\overline{s_{V}}}\right)\right],
$$

where $V_{0}$ is the asymptotic velocity of the fluid, $\alpha$ is its angle, $\Gamma_{C}$ the circle vortex strength, $G_{v}$ the vortex strengths and $s_{V}$ the vortex position in the $s$-plane.

If the action of the boundary is insulated, and skipping the central vortex contribution, we get:

$$
F(s)=\sum_{v=1}^{N_{v}} \frac{\mathrm{i} G_{v}}{2 \pi}\left[-\log \left(s-\frac{b^{2}}{\overline{s_{V}}}\right)\right],
$$

and this leads to an induced velocity from the boundary in the set of points $s$ :

$$
\frac{\mathrm{d} F}{\mathrm{~d} s}=u-\mathrm{i} v=\sum_{v=1}^{N_{v}} \frac{-\mathrm{i} G_{v}}{2 \pi}\left[\frac{1}{s-b^{2} / \overline{s_{V}}}\right] .
$$

When the number of vortices is large, Eq. (21) becomes impractical because the action of the $N_{V}$ images on the $N_{V}$ outside vortices will require an $\mathrm{O}\left(N_{V}^{2}\right)$ work. This problem has been previously noted in the work of Streitlien [23]. They used a fast multipole method (FMM) to get the action of the boundary on the vortices. The next paragraph will explain how to develop a fast imaging procedure which will be shown in Section 7 to be both faster than the FMM evaluation of Eq. (21) and the panel method.

The quantity

$$
\frac{\mathrm{d} F}{\mathrm{~d} s}=\frac{-\mathrm{i}}{2 \pi} \sum_{v=1}^{N_{v}}\left[G_{v} \frac{1}{s-b^{2} / \bar{s}_{v}}\right]
$$

can be rewritten as

$$
\frac{\mathrm{d} F}{\mathrm{~d} s}=\frac{-\mathrm{i}}{2 \pi s} \sum_{k=0}^{+\infty}\left(\sum_{v=1}^{N_{v}} G_{v} a_{k, v}^{\prime}\right) \frac{b^{k}}{s^{k}}
$$




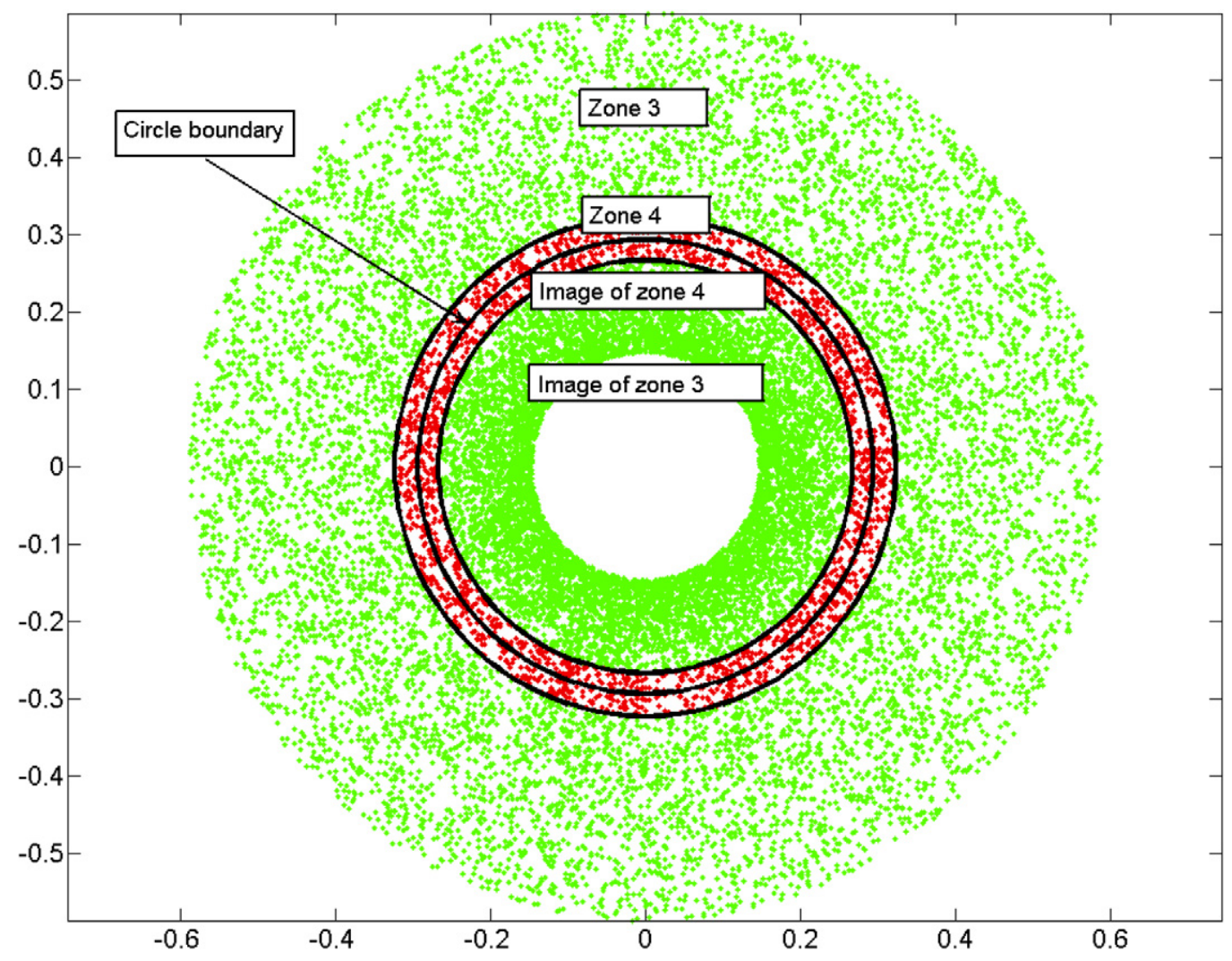

Fig. 4. Illustration of the zones considered in the fast imaging procedure. Zone 3 corresponds to the set of particles where a Laurent's series is used to get the action of the boundary in the particles. Zone 4 corresponds to the particles involving a direct summation of the action of the images of zone 4 and Laurent's series for the action of zone 3. The image of zone 3 and 4 inside the circle are also plotted.

where $a_{k, v}^{\prime}=b^{k} / \bar{s}_{v}^{k}$.

Defining

$$
a_{k}=\sum_{v=1}^{N_{v}} G_{v} \frac{b^{k}}{\bar{s}_{v}^{k}},
$$

we obtain:

$$
\frac{\mathrm{d} F}{\mathrm{~d} s}=\frac{-\mathrm{i}}{2 \pi s} \sum_{k=0}^{+\infty} a_{k} \frac{b^{k}}{s^{k}} .
$$

If this series is truncated at a certain number of terms $N_{\text {trunc }}$, the evaluation will be accurate at least at $\varepsilon_{\text {want }}$ if

$$
N_{\text {trunc }} \geqslant \frac{\log \left|\varepsilon_{\text {want }}^{\prime}\right|+\log (1-X B)-\log (A)-\log \left(G_{v} / 2 \pi\right)}{\log (A)+\log (B)}-1,
$$

where $\varepsilon_{\text {want }}^{\prime}=\varepsilon_{\text {want }} / N_{v}, B=\max _{v \in\left[1, N_{v}\right]}\left|Y_{v}\right|$ and $A=\max |X|$.

Two annulus are considered, depending on the position of the outer vortices: the zone called zone 3 corresponding to the "far" vortices or the vortices placed outside of the circle of radius $11 b / 10$ and the zone 4 which corresponds to the annulus bounded by the circle of radius $b$ and the circle of radius $11 b / 10$. The two zones are depicted in Fig. 4.

In zone 3 the effect of the images of zone 3 and zone 4 are shifted to the origin and the coefficients of the resulting Laurent's series are evaluated using Eq. (25).

In zone 4 , the effect of the image of zone 3 only is shifted to the origin (the Laurent series coefficients are already determined from the previous step) and evaluated using a Laurent series, the effect of the image of zone 4 in zone 4 is evaluated directly. 
It will be shown in Section 7 that the gain in term of computation time is promising compared to vortex panel methods. Concerning every vortices which are placed in zone 3 or generally far from the airfoil or from the circle, the influence of the body is again found as a Laurent's series and therefore simple to evaluate.

\subsection{Single wing in unsteady motion with vortices}

The boundary element problem with no through boundary conditions for unsteady arbitrary motion of a single wing profile is solved exactly as soon as the mapping function is known in the form given in Eq. (4). Milne-Thomson [20] and Couchet [24] gives exact solutions for unsteady arbitrary motion of a single wing with or without vortices. The flow for a pitching airfoil is considered irrotational only in the non-moving inertial lab frame, which enables the determination of the complex potential in this frame. However, the streamfunction exists both in the frame attached to the wing and in the inertial frame. The inviscid potential unsteady motion solution is derived analytically and cast into the form of a Laurent's series only depending on the complex coefficients $\left\{c_{k}\right\}_{k \in \mathbb{N}}$ of Eq. (4). If an unsteady motion with vortices is considered, the complex coefficients coming from the unsteady motion are simply added to the previous ones from the fast imaging procedure and the evaluation in a large number of points is fast. Couchet [24] even derived analytical formulas for deformable wing. The forces are directly calculated from application of the residue theorem.

\subsection{Fast solution in the multicomponent case: steady flow without vortices}

$N_{w}$ circles are considered in a uniform flow. The solution can be expressed in the form of Laurent's series in the circles centers which are also the poles of the mapping function $g$ (Eq. (7)). The general solution for a steady flow around multiple circles without vortices is given as:

$$
F(s)=V_{0} \mathrm{e}^{\mathrm{i} \alpha} s+\sum_{n=1}^{N_{w}}\left[C_{V, n} \log \left(s-s_{\text {center }, n}\right)+\sum_{k=1}^{N_{0}} \frac{g_{n, k} b_{n}^{k}}{\left(s-s_{\text {center }, n}\right)^{k}}\right] .
$$

This solution requires the computation of $N_{w} \times N_{0}$ complex coefficients $g_{n, k}$ to ensure the no through boundary condition and $N_{w}$ coefficients $C_{V, n}$ to satisfy, if needed, the Kutta conditions at all trailing edges of the airfoil sections.

Recent work from Crowdy [25] and its practical implementation [26] proved the existence of an analytic solution of this problem. However we will here use an iterative procedure. Williams [27] showed that the following procedure was convergent for the case of two circles. Prosnak [22] expressed also the multi circle flow in this form; however he found the coefficients differently. It was not determined which algorithm is faster.

The following steps are used: image once in each circle the external uniform flow, evaluate the velocity in each trailing edge. Find the solution of a linear system $N_{w} \times N_{w}$ to nullify the velocity at the trailing edges. Introduce in each circle center vortices with strength $C_{V, n}$. Take each circle independently, the outer flow from the point of view of the circle $N_{c}$ in its complex velocity form is (the uniform asymptotic flow does not need to be imaged again)

$$
\frac{\mathrm{d} F_{N_{c} \text { out }}}{\mathrm{d} s}(s)=\sum_{n \neq N_{c}} \sum_{k=1}^{N_{0}} \frac{d_{n, k} b_{n}^{k}}{\left(s-s_{C, n}\right)^{k}} .
$$

With the help of Milne-Thomson [20], the form of the induced velocity that this circle should provide to satisfy the no through boundary condition is:

$$
\frac{\mathrm{d} F_{N_{c} \text { in }}}{\mathrm{d} s}(s)=-\sum_{n \neq N_{c}} \sum_{k=1}^{N_{0}} \frac{\bar{d}_{n, k} b_{n}^{k}}{\left(b_{N_{c}}^{2} /\left(s-s_{C, N_{c}}\right)+\bar{s}_{C, N_{c}}-\bar{s}_{C, n}\right)^{k}} \cdot \frac{b_{N_{c}}^{2}}{\left(s-s_{C, N_{c}}\right)^{2}} .
$$

Two solutions are possible:

On one hand, evaluate this expression on $2 P$ points on the boundary, use an FFT to make the synthesis and obtain the Laurent's series coefficients for each circle, add these coefficients to the one found at the previous time step and iterate again. 
Another choice is to use the coefficients found analytically when Eq. (30) is rewritten as

$$
\frac{\mathrm{d} F_{N_{c} \text { in }}}{\mathrm{d} s}=-\sum_{n \neq N_{c}} \sum_{k=1}^{N_{0}} \frac{\bar{d}_{n, k} b_{n}^{k}}{\left(\bar{s}_{C, N_{c}}-\bar{s}_{C, n}\right)^{k}\left(1+b_{N_{c}}^{2} /\left(\left(\bar{s}_{C, N_{c}}-\bar{s}_{C, n}\right)\left(s-s_{C, N_{c}}\right)\right)\right)^{k}} \cdot \frac{b_{N_{c}}^{2}}{\left(s-s_{C, N_{c}}\right)^{2}},
$$

with

$$
\left|\bar{s}_{C, N_{c}}-\bar{s}_{C, n}\right|>b_{N_{c}} \text { and }\left|s-s_{C, N_{c}}\right| \geqslant b_{N_{c}},
$$

and therefore for any point $s$ outside or on the $N_{c}$ th circle:

$$
\frac{1}{\left(1+b_{N_{c}}^{2} /\left(\left(\bar{s}_{C, N_{c}}-\bar{s}_{C, n}\right)\left(s-s_{C, N_{c}}\right)\right)\right)^{k}}=\sum_{p=0}^{+\infty} \frac{(k)_{p}}{p !}(-1)^{p} \frac{b_{N_{c}}^{2 p}}{\left(\bar{s}_{C, N_{c}}-\bar{s}_{C, n}\right)^{p}\left(s-s_{C, N_{c}}\right)^{p}},
$$

where $(k)_{p}$ corresponds to the Pochhammer symbol of $k$.

Inserting this quantity into Eq. (31) gives:

$$
\frac{\mathrm{d} F_{N_{c} \mathrm{in}}}{\mathrm{d} s}=-\sum_{n \neq N_{c}} \sum_{k=1}^{N_{0}} \sum_{p=0}^{+\infty} \frac{(k)_{p}}{p !}(-1)^{p} \frac{\bar{d}_{n, k} b_{n}^{k} b_{N_{c}}^{p}}{\left(\bar{s}_{C, N_{c}}-\bar{s}_{C, n}\right)^{p+k}} \frac{b_{N_{c}}^{p}}{\left(s-s_{C, N_{c}}\right)^{p}} \cdot \frac{b_{N_{c}}^{2}}{\left(s-s_{C, N_{c}}\right)^{2}},
$$

and

$$
\frac{\mathrm{d} F_{N_{c} \text { in }}}{\mathrm{d} s}=\sum_{p=0}^{+\infty}\left[\sum_{n \neq N_{c}}\left[\sum_{k=1}^{N_{0}} \frac{(k)_{p}}{p !} \frac{(-1)^{p+1} \bar{d}_{n, k} b_{n}^{k} b_{N_{c}}^{p}}{\left(\bar{s}_{C, N_{c}}-\bar{s}_{C, n}\right)^{p+k}}\right]\right] \frac{b_{N_{c}}^{p+2}}{\left(s-s_{C, N_{c}}\right)^{p+2}} .
$$

The contribution from the $N_{c}$ th circle at this iteration will be to add a pole in the center of this circle and the coefficients of the Laurent series of this contribution are directly given by

$$
\tilde{D}_{p+2, N_{c}}=\sum_{n \neq N_{c}}\left[\sum_{k=1}^{N_{0}} \frac{(k)_{p}}{p !} \frac{(-1)^{p+1} \bar{d}_{n, k} b_{n}^{k} b_{N}^{p}}{\left(\bar{s}_{C, N_{c}}-\bar{s}_{C, n}\right)^{p+k}}\right] \text {. }
$$

In practice this series will be truncated. The coefficients are then added to the coefficients obtained at the previous step for the same circle. The number of needed coefficients for certain accuracy in the iterative solution at each time step can be estimated by the same method as in Eq. (27).

This procedure will necessitate a small number of coefficients, as far as all circles have the same radius and are not placed too close to each other. The case of different circles with radius of different orders of magnitude can lead to troubles especially when one of the small circle is very close to one of the big one. Adding an extra pole at exactly the imaging point of the center of this small circle improves the convergence.

If a Voith Schneider or its wind turbine counterpart, an $H$ rotor section, is considered, the wings are conveniently far from each other. It has been estimated that 8 iterations with 16 coefficients lead to a maximum error of $10^{-6}$. In Fig. 5, a plot of the evolution of the error with respect to the number of iterations in a typical $H$ rotor section is provided. When wings are closer to each other, an accuracy of $10^{-6}$ can be reached in 15 iterations and 32 terms in the series.

Typical streamlines for the case of 8 circles are plotted in Fig. 6. The velocity is deduced from Eq. (17). If the induced velocity, due to the boundaries, needs to be computed in many points, the truncation order and fast evaluation is simple considering the form of Eq. (17) for the mapping and Eq. (28) for the potential flow solution.

\subsection{Multicomponent case: asymptotic steady flow with vortices}

The case of steady asymptotic flow with vortices has to be investigated in more details. We will here refer to the two zones we have identified in the fast imaging procedure (see Fig. 4).

The form of Eq. (28) is still valid if all vortices are in zone 3 for each of the circles. The fast imaging procedure is performed for each circle. The large number of coefficients due to the vortices action is only evaluated once at the beginning of the procedure. After this step 32 coefficients have to be considered as they just need to correct the action of the other wings. The only significant computation time will be found in the first iteration. 


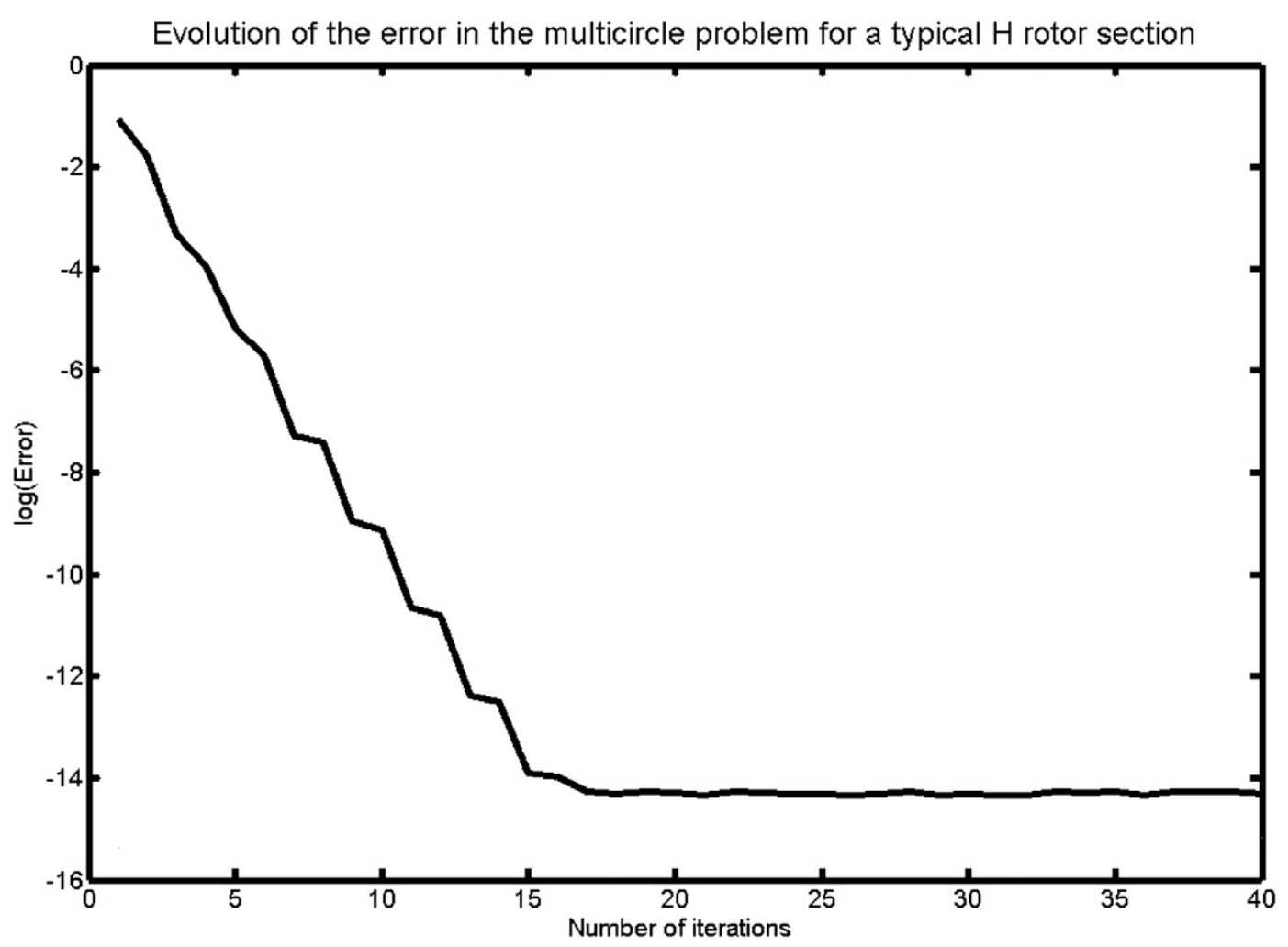

Fig. 5. Log of the error in the multielement boundary problem versus the number of iterations for a typical $H$ rotor section represented by three circles. The error is evaluated as the maximum, over all circles, of the scalar product of the velocity by the normal to the circle. 1000 points are placed in each circle.

When vortices are found in zone 4 for one circle (see Fig. 7), these vortices are removed when the induced velocity from the outer flow is evaluated. They are kept as the reaction of this circle on the others and expanded in zone 3 . The vortices are added for the evaluation of points close to the critical circle at the end of the computation. This is shown in Fig. 7 for the degenerated case of only one vortex in zone 3 for four circles with one vortex close to the second circle.

\subsection{Multicomponent case, unsteady flow with vortices}

Although the solution for an unsteady flow around multicomponent airfoil is not detailed here, such a solution can be obtained by exploiting the exact solution known for one single wing in unsteady motion which can be found in Couchet [24]. The procedure applied from Eqs. (4.14)-(4.21) is applicable.

As a conclusion of this paragraph, we will summarize some advantages of finding the mapping function in the Eqs. (4) and (7) and the flow solution in form Eq. (28).

First, the solution of many circle flows can be obtained to any degree of accuracy at a limited computational time. This can be used to generate test cases for multicomponent section where few exact solutions exist. The method can also be applied to inverse design procedures as it provides a parameterization of the full multicomponent section. Furthermore, the lift coefficient is obtained by application of the residue theorem, in the ideal flow case, both for the single and the multicomponent section.

The implementation of the method is facilitated by the inclusion of functions from complex numbers to complex numbers into standard programming tools like Matlab or C99.

The formalism of Laurent's series is ideally suited to be included into fast multipole methods for particle flow simulation. The development of the vortex particle procedure can be followed in the many circle plane, simplifying 


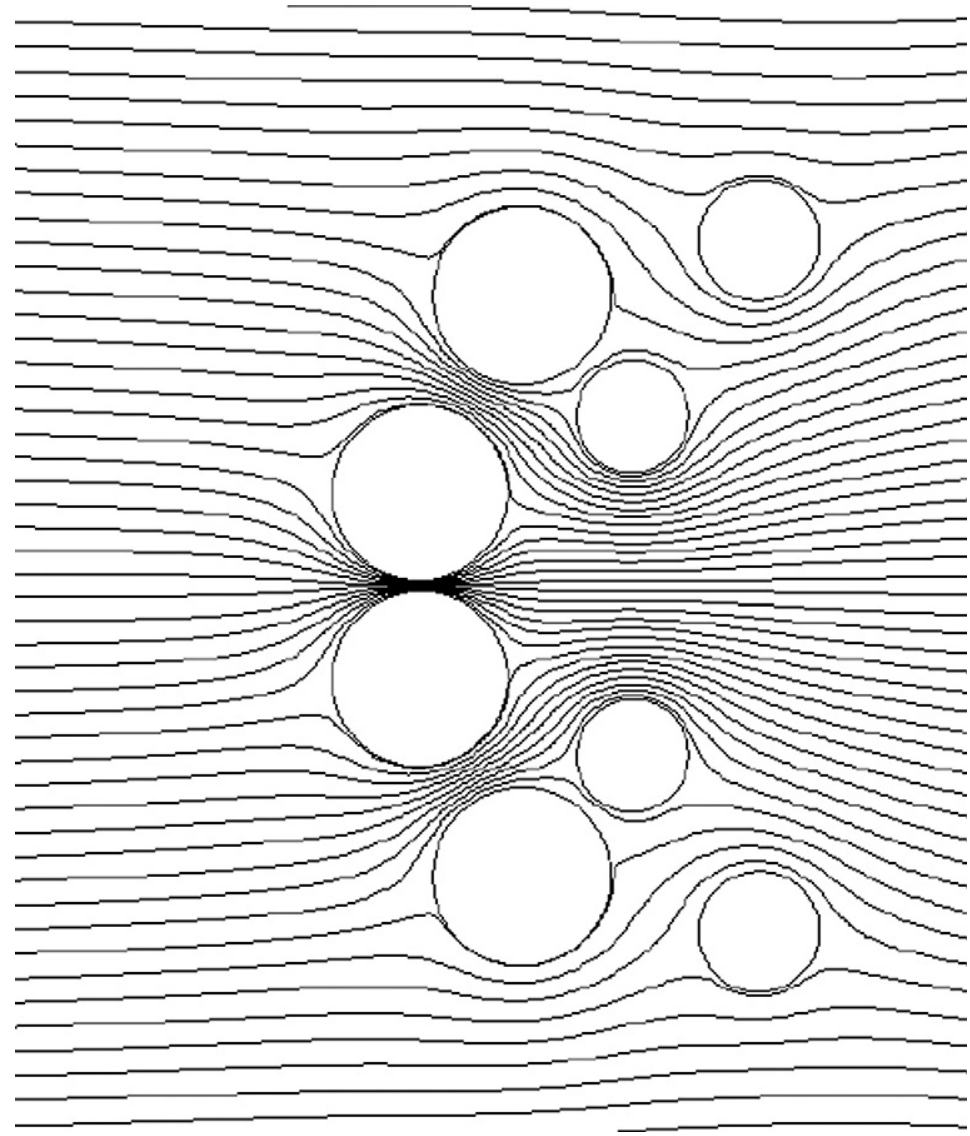

Fig. 6. Potential flow solution for eight circles placed in a uniform asymptotic flow. The obtained accuracy is $10^{-6}$. A polar grid is used for the streamline plot.

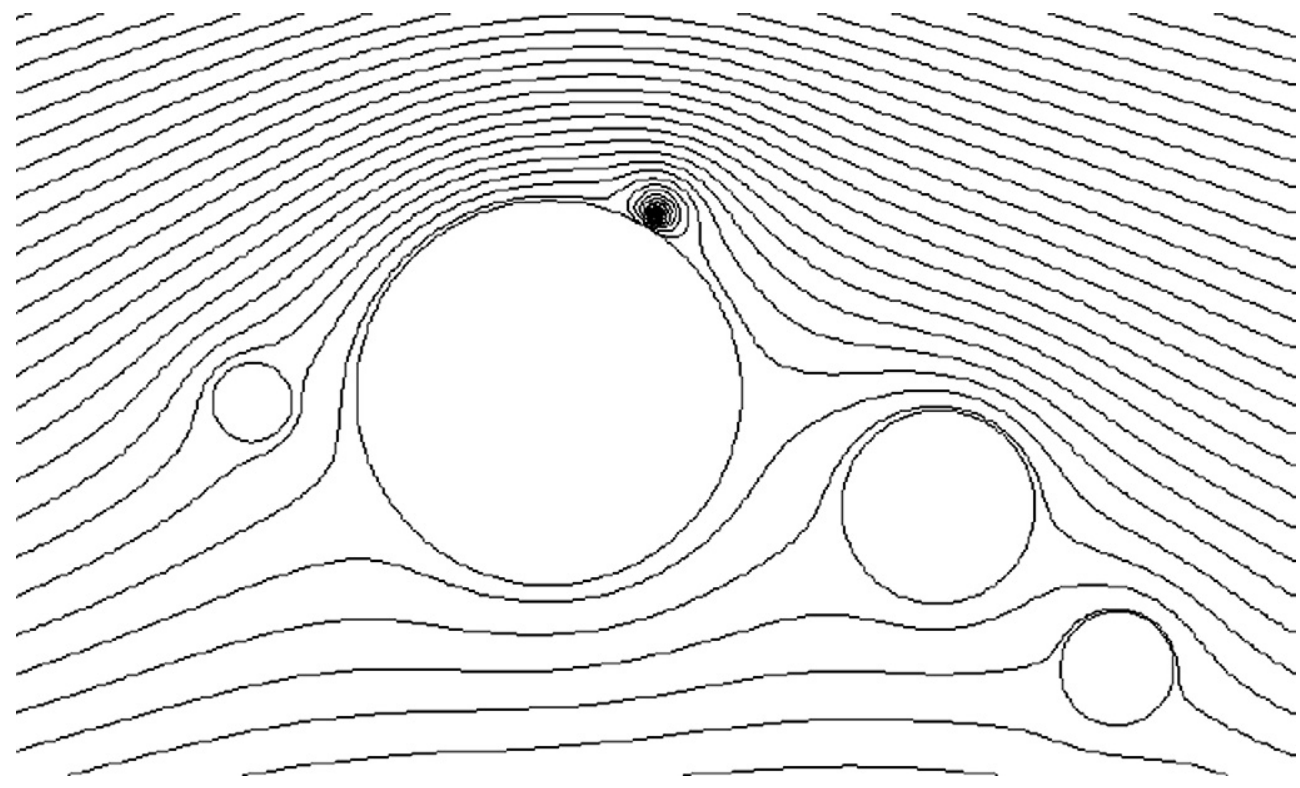

Fig. 7. Four circle solution in asymptotic uniform flow with one logarithmic singularity usually representing a vortex in simple vortex particle theories. The singularity is placed very close to the main element surface to check the accuracy of the potential flow solution. The accuracy is retained. 
and improving the numerics. The Routh's corrections [28] should be implemented in the convection step facilitated by an appropriate series truncation in the first and second derivative of the mapping function,

The method is not making use of control points as in the panel method case. For many circles flows, the method usually necessitates 64 coefficients for a very accurate tangential velocity evaluation without the non-physical harmonics due to edges effects.

Additionally, structured meshes can be obtained when evaluating isolines of the potential and streamfunction by taking the real and imaginary part of Eq. (28).

The speed of the method, to obtain the many circles flows, scales as $\mathrm{O}\left(N_{W}^{2} N_{0}^{2}\right)$ to the leading order with $N_{0}$ equal to 64. It is faster than the standard panel methods which scale as $\mathrm{O}\left(N_{W} N_{\text {Panel }}\right)^{3}$ or at least $\mathrm{O}\left(N_{W} N_{\text {Panel }}\right)^{2}$ [19]. Typical panel numbers for accurate particle methods are in the range of $N_{\text {Panel }}=160$ to 800 .

A more critical point is that the action of the multiple boundaries in the vortex particles is faster evaluated than if a panel method is used. In a sense, the multipole solution both for the mapping and the flow provides the solution on the boundaries plus a synthesis for the fast evaluation in a large number of points. This advantage will be demonstrated in Section 7.

The mapping enables for semi analytical and analytical solutions. Many solutions exist for complicated fluid flows as far as the mapping function can be accessed. In the case of linear or periodic cascades, by a first mapping, all blades go to one, which is then mapped into a circle leading to an exact solution as explained in Lewis [18].

Moreover, for the setup and configuration of flow actuators, it is important to provide good and fast results; the present method might help to achieve this goal.

\subsection{Overall mapping methodology}

Prosnak [22] has previously derived a numerical method to obtain the conformal mapping of multicomponent sections using Laurent's series. However, the method developed here employs an assembly of previous methods with improvements and has therefore been developed and benchmarked using previous work. Although, more recent mapping techniques might be more general [10,29], the Theodorsen method [4] will be used with the help of fast Fourier transform as defined in Ives [5]. In the present work, the procedure is made flexible enough to accept a certain number of corners.

The evaluation of the Laurent's coefficient is detailed. Concerning the multielement section, the method is similar to that of Halsey [9] with improvements for the treatment of inverse transformations which have been found to be a critical bottleneck for rapidity.

From the authors' experience it has been found that each of the following steps are crucial to provide a robust mapping algorithm, that is why they are all described here. New findings concern the inversion of the mapping applied to multicomponent mapping, the ability of the corner remover to expand considerably the vast variety of shapes which can be mapped to circles and finally the synthesis using Laurent's series.

\section{Building of the mapping function: Laurent's series expansion for a single wing}

This procedure will be detailed in the six subsequent steps. The aim is to find $N$ Laurent's series coefficients, $\left\{c_{k}\right\}_{k \in \mathbb{N}}$, the radius of the corresponding circle $b$ and the parameter $\sigma$ representing the wing given by a set of points converted into complex numbers.

Step 1: scaling. First, the initial points of the airfoil are interpolated into $2 N$ points. At this stage the airfoil is scaled as desired. Additionally the airfoil is centered on the origin and a rotation is used to place the trailing edge along the real axis. This transformation reads:

$$
z_{N}=\left(z+z_{T}\right) \mathrm{e}^{\mathrm{i} \phi} .
$$

Care should be taken during the interpolation step to ensure a smooth representation of the wing, unless the wing has corners. Smoothing procedure, using splines or lines between points, will have an important effect on the pressure distribution derived from this model (see Section 7).

Step 2: Inverse Karman Trefftz transformation: corner remover. The second step requires the trailing edge angle identification $\tau$ and estimation of the radius of curvature $R$ at the leading edge point $z_{\mathrm{TE}}$. An inverse Karman Trefftz transform is applied to the points $z$ as: 


$$
\begin{aligned}
& z^{\prime}=\beta \frac{z_{0}-\alpha z_{1}}{1-\alpha}, \\
& \alpha=\left(\frac{z_{N}-z_{0}}{z_{N}-z_{1}}\right)^{\beta} \mathrm{e}^{\mathrm{i} 2 \pi r \beta},
\end{aligned}
$$

where $r$ is an integer typically 0 or $1 . \beta=\pi /(2 \pi-\tau)$ and the two constants $z_{0}$ and $z_{1}$ are given by $z_{0}=z_{\mathrm{LE}}+R / 2$ where $z_{\mathrm{LE}}$ is the trailing edge point and $z_{1}=z_{\mathrm{TE}}$.

The aim of this transformation is to smooth all trailing edges corners of the original shape. This transform is multiply valued and the suitable branch have to be identified.

It should be stressed that the parameter $r$ in Eq. (38b) jumps from the value 0 to -1 if a point is found inside the domain bounded by the mid chord and the line joining the two points $z_{0}$ and $z_{1}$. With this in mind, the Inverse Karman Trefftz transformation can be easily used for a large number of points efficiently.

Step 2 bis methodic corner remover. If the airfoil presents other discontinuities, it is possible to iterate this mapping by placing the parameter $z_{1}$ exactly at the remaining discontinuities. If the angle is pointing towards the inside of the profile, the value $\tau$ has to be adjusted to stay inside $[0,2 \pi]$. The value for the parameter $z_{0}$ is chosen as the intersection between the bisector of the angle and the opposite side of the curve. If it is impossible to reach this point without intersecting the curve, the point, which maximizes the distance between the trailing edge and the circle, should be chosen. The point is then displaced in the direction pointing to $z_{1}$ by the corresponding radius of curvature.

When all corners are removed, a shape similar to an ellipse can appear. Then one last reverse Joukowsky mapping is applied to transform this ellipse onto a quasi circle. This last procedure only needs to be applied for peculiar cases such as the mapping of flat back airfoils, airfoil with spoiler or gurney flaps (see Section 8). For usual airfoil sections, one first inverse Karman Trefftz is enough.

Step 3: rescaling. The third step involves a simple translation and rotation

$$
z^{\prime \prime}=\left(z^{\prime}+z_{\text {center }}^{\prime}\right) \mathrm{e}^{-\mathrm{i} \chi},
$$

to ensure that the obtained $z^{\prime}$ curve is a centroid centered at the origin and that the trailing edge is on the real axis. The centroid is found by splitting the points and the origin into triangles and by assessing the center of gravity from the triangles.

In the $z^{\prime \prime}$ plane all points are decomposed into the form

$$
z^{\prime \prime}=\exp \left(\ln r_{i}+\mathrm{i} \theta_{i}\right)
$$

where $\left(\psi_{i}, \theta_{i}\right)$ is given by

$$
\begin{aligned}
& \ln r_{i}=\operatorname{Re}\left[\ln z^{\prime \prime}\right], \\
& \theta_{i}=\operatorname{Im}\left[\ln z^{\prime \prime}\right] .
\end{aligned}
$$

The convergence of the foregoing method should be checked. According to Ives [5], convergence is ensured if (but not only if)

$$
\left|\frac{\mathrm{d}(\ln r)}{\mathrm{d} \theta}\right|<0.2954976 .
$$

Step 4: Theodorsen transform. The fourth transformation due to Theodorsen [4], transforms the $z^{\prime \prime}$ plane to the final $s$-plane. It is given in the form of a series expansion truncated with $N$ terms:

$$
z^{\prime \prime}=s \cdot \exp f(s),
$$

where $f(s)=\sum_{1}^{N} C_{n}^{0} b^{n} / s^{n},\left\{C_{n}^{0}\right\}_{n} \geqslant 1$ are complex numbers chosen to preserve the identity at infinity. In the $s$-plane, the equation of the circular boundary is given as:

$$
s=b \mathrm{e}^{\mathrm{i} \eta}
$$

Substituting Eq. (44) into Eq. (43) at the circle boundary gives

$$
\ln r+\mathrm{i}(\theta-\eta)=\ln b+\sum_{1}^{N} C_{n}^{0} \mathrm{e}^{-\mathrm{i} n \eta} .
$$


Fast Fourier transform techniques may now be used as described in Ives [5]: the angles $\eta$ for $2 N$ points are equally spaced in the circle from the trailing edge

$$
\eta_{k}=2 \pi(k-1) / 2 N \quad \text { with for } 1 \leqslant k \leqslant 2 N .
$$

An iteration procedure is then necessary, the initial values of the coefficients $\left\{C_{n}^{0}\right\}_{n} \geqslant 1$ and $\ln b$ are set to zero. The vector $Y$ is introduced, as

$$
\begin{aligned}
& y_{1}=0, \\
& y_{j+1}=\mathrm{i} \bar{C}_{j}^{0} / 2 \quad \text { for } 1 \leqslant j \leqslant N-1, \\
& y_{N+1}=0, \\
& y_{2 N-j+1}=\bar{y}_{j+1} \quad \text { for } 1 \leqslant j \leqslant N-1 .
\end{aligned}
$$

The new value of the vector $\theta$, will be deduced from $\eta$ and $Y$ by the Fourier transform evaluated using FFT:

$$
\theta=\eta+\varepsilon \quad \text { with } \varepsilon=\operatorname{IFFT}(2 N Y),
$$

where IFFT stands for Inverse Fourier transform. The expression $(\ln r)_{k}$ is then reevaluated at the points $\theta_{k}$. With these values, the value of $Y$ is calculated after another FFT:

$$
Y=F F T(\ln r),
$$

and from $Y$ the coefficients $\left\{C_{n}^{0}\right\}$ are evaluated by:

$$
C_{j}^{0}=2 \bar{y}_{j+1} \quad \text { for } 1 \leqslant j \leqslant N .
$$

This process is iterated until the satisfactory accuracy is reached. 8 iterations are necessary.

Step 5: Coming back from the circle to the wing. The radius of the mapped circle is obtained by

$$
b=\mathrm{e}^{\operatorname{Re}\left[y_{1}\right]} .
$$

When the coefficients are known, $2 N+2$ angles $\eta$ are chosen uniformly on the circle. The transformation, from the circle to the airfoil, from the $s$-plane to the $z$ plane, is defined by:

$$
\begin{aligned}
& z^{\prime \prime}=s \cdot \exp f(s), \\
& z^{\prime}=z^{\prime \prime} \mathrm{e}^{\mathrm{i} \chi}-z_{\text {center }}^{\prime}, \\
& z_{N}=\frac{z_{1}-\beta z_{0}}{1-\alpha^{\prime}},
\end{aligned}
$$

with $\alpha^{\prime}=\left(\left(z^{\prime}-\beta z_{1}\right) /\left(z^{\prime}-\beta z_{0}\right)\right)^{1 / \beta}$.

If more than one Karman Trefftz has been used, the transformations are iterated. The last transform is:

$$
z=z_{N} \mathrm{e}^{-\mathrm{i} \phi}-z_{T} \text {. }
$$

Step 6: Synthesis: wanted coefficients. The Laurent's series coefficients are obtained by a last inverse FFT on the $2 N+2, z$ points

$$
\begin{aligned}
& c_{n}^{\prime}=\operatorname{IFFT}(z), \\
& \sigma=c_{1}^{\prime} .
\end{aligned}
$$

To accommodate the two rotations, the $N$ coefficients retained are modified as

$$
c_{n}^{\prime \prime}=c_{n+1}^{\prime} \mathrm{e}^{\mathrm{i} n(\chi-\phi)},
$$

for $n \in[1, N]$.

Gibbs phenomenon can appear due to interpolation errors, and then it can be useful to filter higher harmonics with

$$
c_{n}=\sin c\left(\frac{n \pi}{N+1}\right) b^{n} c_{n}^{\prime \prime},
$$


where $\sin c(x)=\sin (x) / x$ is the sine cardinal function and $N$ is the number of terms in the truncated complex Fourier series. The trailing edge angle can be found by analyzing the derivative of the mapping or by tracking the corner point from the start of the process.

All the parameters needed for evaluation of the mapping function in Eq. (4) are now defined. In practice, a small $N$ is needed. For smooth wing sections, a value of $N=64$ is enough to provide a relative error of $10^{-5}$ in the mapped shape. In the case of flat back airfoils, gurney flaps and spoilers, the number of terms needs to be increased. This will be studied in Section 8.

The number of operations for this procedure scales as $4 C N \log (2 N)$ where $C$ is typically around 8 . Thus this method will not affect the rapidity of the flow solver in the comparison to panel methods for particle method flows.

The rapidity of such a transformation becomes more pronounced whenever the geometry is changing, for instance in animal locomotion or deformable wings. In those cases this mapping method can be preferred to panel methods due to its ability to use one universal geometry for all calculations. This has been pointed out by Couchet [24] for the study of deformable structures.

\section{Laurent's series expansion of multicomponent sections}

The Laurent series expansion of the general multicomponent section can be considered as similar to the previous single component mapping. The first step is a set of $N_{w}$ translation and rotation with $N_{w}$ inverse Karman Trefftz maps described in Eq. (38). After that, one wing is chosen and the previous method is also applied to transform the quasi circle obtained into one exact circle. However, all the other wing sections will be affected by this transformation. To follow the procedure it is important to possess an inverse form of the Theodorsen transform, which according to Eq. (43) writes:

$$
z=s \cdot \exp f(s)
$$

with $f(s)=\sum_{1}^{N} C_{n}^{0} b^{n} / s^{n}$ where the coefficients $\left\{C_{n}^{0}\right\}$ are known.

This can be expanded into the power series

$$
z=s \sum_{l=1}^{N} \frac{k_{l}}{s^{l}},
$$

where $k_{l}$ is found recurrently by

$$
\begin{aligned}
& k_{0}=1, \\
& k_{l}=\frac{1}{l} \sum_{p=1}^{l} p k_{l-p} C_{p}^{0} b^{p} .
\end{aligned}
$$

To obtain in this context a quick evaluation of the inverse transform the procedure detailed in Section 3 is used. In this form the amount of time to perform the inverse procedure becomes negligible: a few ms in a normal computer.

The inverse of the transformation should be rigorously applied to each quasi circle comprising the first one. However, the result for this first circle will be to produce a circle of radius $b_{1}$ in virtue of the algorithm described in Section 3. Thus, all the points from this wing are replaced by a circle with uniform angles distribution. Only the trailing edge position is tracked. The other quasi circles are inversed using the fast inverse transform.

The next steps involves iteration of this quasi-circle-to-circle mapping to all quasi circles and repetition of this process until the shapes are close enough to circles. Then the center and radius of the final circles are found and the trailing edge corresponding angles are known.

To get the synthesis of the mapping, $2 N$ points are uniformly distributed on each circle. Once all the transforms are applied, $2 N$ points are obtained on each wing. To derive the Laurent's series decomposition, it suffices to use the inverse Fourier Transform and cancel all terms, which are corresponding to non-convergent ones (i.e. with power more than $N$ ).

All the rotations have also been counted and the two steps described by Eq. (58) and the filter step in Eq. (59) are applied.

8 iterations are needed. One iteration being defined as a whole round: each circle has been mapped to an exact circle once. This method is scaling as $4 C^{\prime} N N_{w} \log \left(2 N N_{w}\right)$ and $N$ is typically of the order of 64 to obtain a relative 
Table 1

CPU time comparison for different methods for the boundary action on 10000 particles

\begin{tabular}{|c|c|c|c|c|c|c|c|c|}
\hline \multirow[b]{3}{*}{ CPU time in seconds } & \multicolumn{5}{|c|}{ Panel method } & \multicolumn{3}{|l|}{ Image method } \\
\hline & 160 panels & 400 panels & 600 panels & 800 panels & 1200 panels & Direct imaging & FMM imaging & Fast imaging \\
\hline & 1.33 & 3.33 & 4.98 & 6.65 & 9.98 & 5.31 & 1.3 & 0.3 \\
\hline
\end{tabular}

accuracy of $10^{-5}$. This mapping combined with the many circles flow solution given by Eq. (28), results in a fast solution of the multiple body boundary element problem.

\section{Fast imaging and panel methods with vortices}

To illustrate the advantage of the present method, it is important to compare it against a typical boundary element method, the vortex panel method [17].

A circle is considered to make a comparison between the flow solver and existing boundary element method. 10000 particles are placed uniformly around it in an annulus between the circle with a radius 1 and another circle with a radius of 2 . Although no particles are placed on the boundary they are placed arbitrarily close to it.

We will compare in Table 1 the results in terms of computational time of the boundary action on the vortices in Eq. (22).

On one hand, we will consider panel methods with $N_{\text {panel }}=160$, the lowest number of panels used for vortex particle simulation [30], $N_{\text {panel }}=400$ used in Ramachandran [19], $N_{\text {panel }}=600$ for Walther [31], $N_{\text {panel }}=800$ and $N_{\text {panel }}=1200$.

On the other hand, the image method is employed. The first implementation concerns the straight forward application of Eq. (22), the second uses a Fast multipole method applied to the images (FMM imaging) and the third run employs the Fast imaging procedure described in Section 3. In the two approximate methods (FMM imaging and Fast imaging), the tolerance has been set to $10^{-6}$.

From Table 1, for a choice of $N_{\text {panel }}=160$, which gives substantial edge effects, to avoid, particularly in high Reynolds number flows, the Fast imaging is 4.4 times faster. For more standard values using from $N_{\text {panel }}=400$ to $N_{\text {panel }}=800$, the Fast imaging is respectively 11 to 22 times faster.

The Direct imaging procedure, although it is exact, corresponds to the same computational time as a panel method with 630 panels.

The Fast imaging procedure is 4.4 times faster than the FMM imaging. This is probably due to the long time required to build the necessary quad-trees which are not fully used as the points of evaluation are outside the circle.

If much less particles are considered, for instance 1000 points, the fast imaging takes $0.052 \mathrm{~s}$ whereas the panel method with 160 panels necessitates $0.22 \mathrm{~s}$.

In fact the comparison in Table 1 should be even more advantageous for the fast imaging procedure if the solution and setup of the linear system for the boundary vorticity would have been included in the CPU time. Only flat panel methods are considered which are supposed to be the fastest to be evaluated.

Ramachandran [19] proposed an alternative to combine multipole expansions and flat panel methods.

We conclude that the mapping method consists in a substantial improvement to panel methods for particle simulation as it can provide the action of the boundaries on vortices in a much more tractable time than standard panel methods. The degree of accuracy can also be increased up to computer precision. The present method consists in a substantial improvement as vortex methods generally necessitates 1000 to 10000 time steps to operate. Furthermore, the fast imaging procedure is very easy to code.

\section{Mapping perspectives/examples}

\subsection{Single component steady flow: comparison with panel method results}

The S809 section has been mapped and placed into a uniform flow to present both the accuracy of the mapping procedure and the accuracy of the deduced boundary element method. From Eq. (19), the velocity is deduced. The cor- 


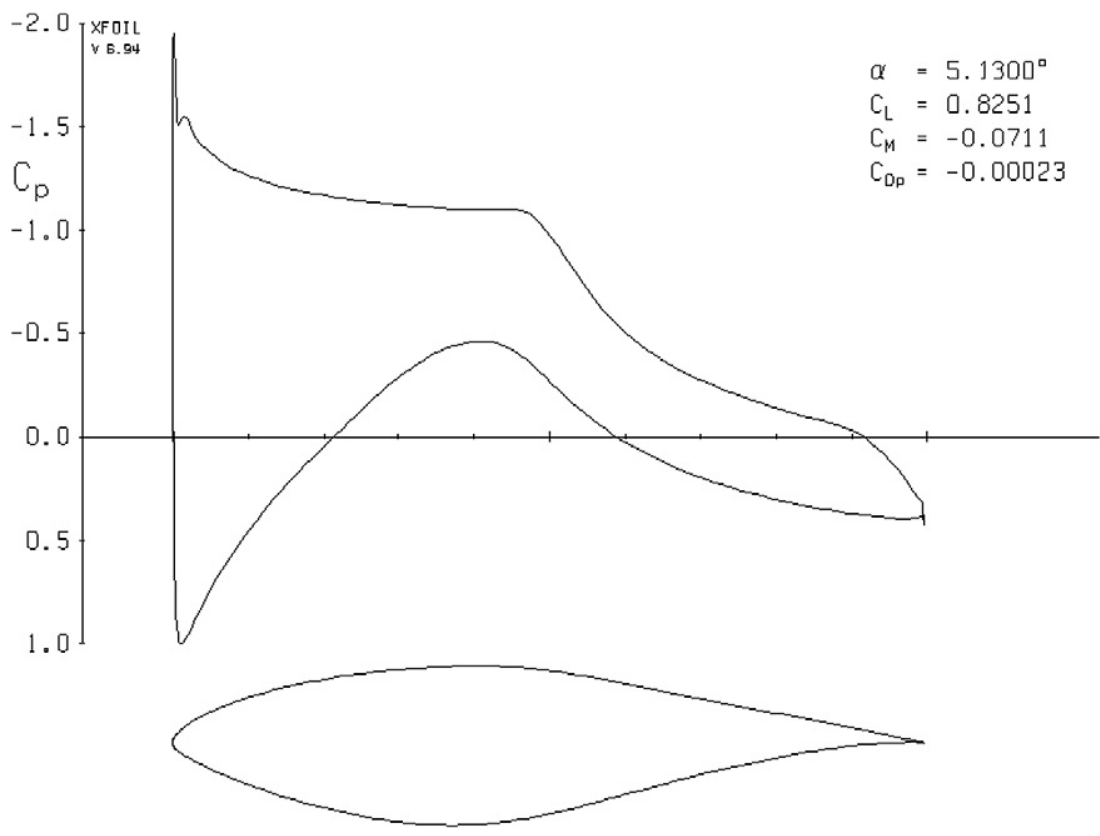

Fig. 8. Normalized pressure coefficient (see Eq. (63)) obtained by the linearly varying vorticity panel code XFOIL using 280 panel for the S809 wing section. The angle of attack is $-5.13^{\circ}$.

responding pressure coefficient will be given by:

$$
C_{P}=\frac{p-p_{\infty}}{1 / 2 \rho V_{0}^{2}}
$$

with $\left(p-p_{\infty}\right) / \rho=-\left(|V|^{2}-\left|V_{0}\right|^{2}\right) / 2$, and thus

$$
C_{P}=1-|V|^{2} /\left|V_{0}\right|^{2} \text {. }
$$

The results obtained with the XFOIL [32] linear vorticity panel method with 280 panels (see Fig. 8) are compared with the results of the present algorithm (see Fig. 9). 64 coefficients are used in the mapping series and 1000 points are used for the plotting. The pressure is in very good agreement with XFOIL results. Furthermore, the results do not show any oscillation even with this huge number of points compared to the number of terms in the series. By application of the residue theorem, the lift coefficient is found as 0.831 which compares well with the XFOIL value of 0.825 . The pitching moment is given as -0.072 , which is in good agreement with the XFOIL value of -0.0711 . Fig. 10 shows the good sensitivity to the shape interpolation and efficiency of the filtering described in Eq. (59). This is the same S809 airfoil at the same angle of attack as previously but without filtering and with a linear interpolation instead of the cubic splines.

64 coefficients seem to be enough to represent a wing shape accurately. However, a wide number of classical wing sections have been derived by the Eppler code [6]. This program is exactly finding the coefficients in the mapping function from a desired velocity distribution at different angles of attacks. It is thus not surprising that only 64 coefficients are enough for most sections.

\subsection{Single airfoil mapping coefficients}

The NACA0018 section is defined from polynomials functions of the position along the real axis. The 64 coefficients for the NACA0018 with unit chord are given in Appendix A to provide some more realistic cases than the Joukowsky profiles for vortex method using conformal mapping. 


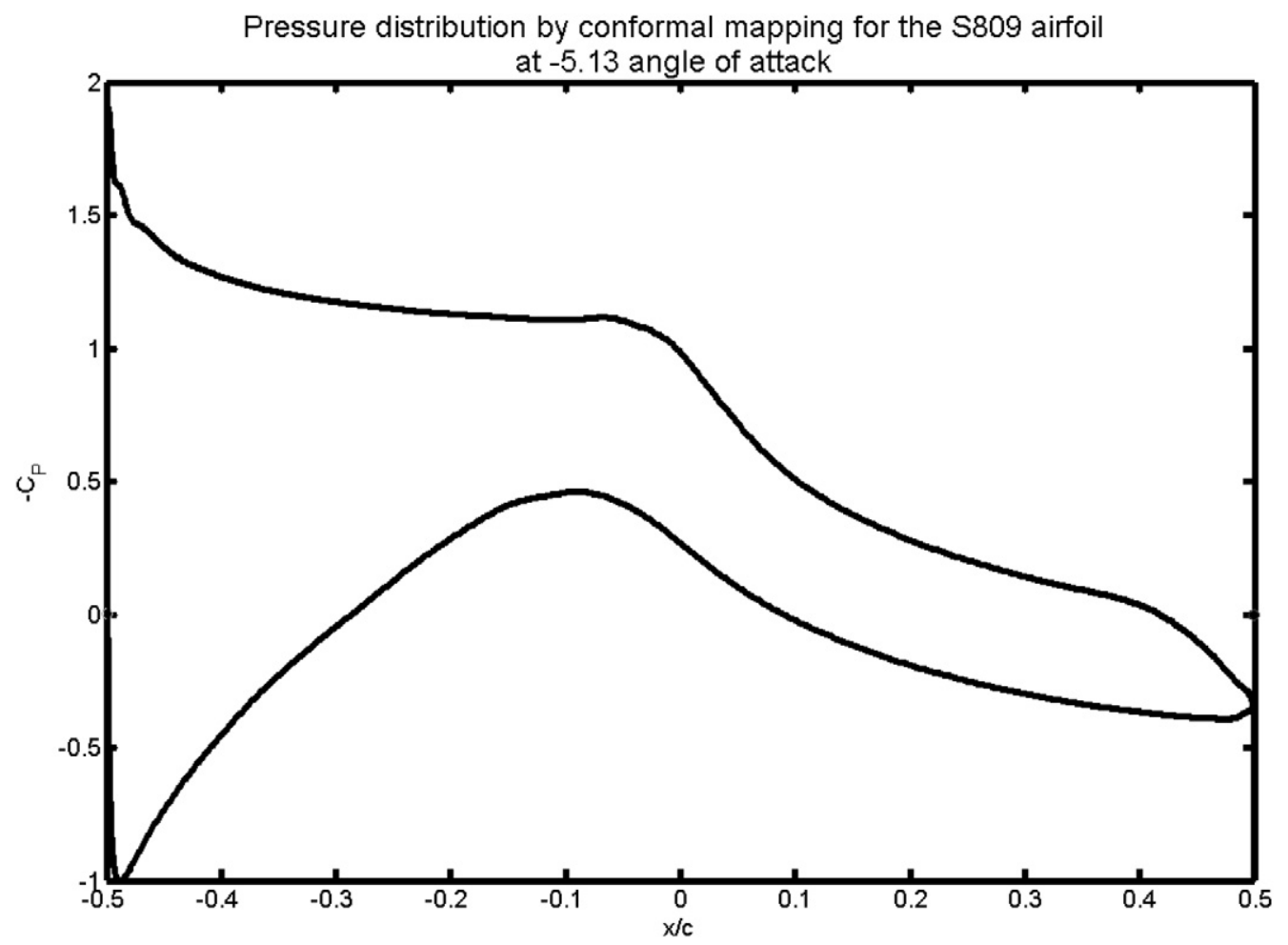

Fig. 9. Normalized pressure coefficient obtained for the same S809 section using 64 coefficients and the mapping procedure. The Lift and pitching moment coefficient are then deduced exactly from the residue theorem.

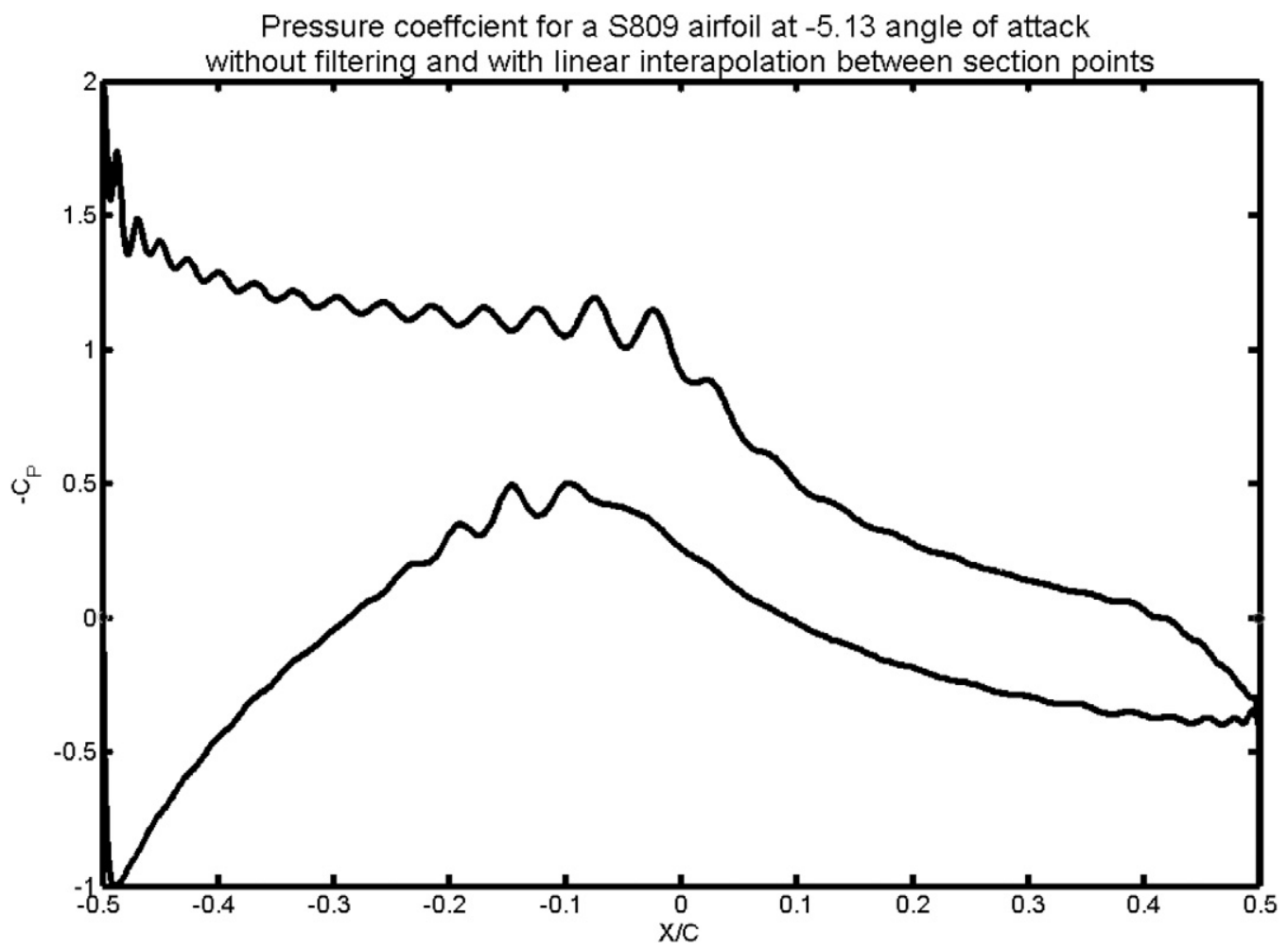

Fig. 10. Normalized pressure coefficient obtained if the surface is discretized by lines instead of splines and without filtering of Eq. (59). 
Corner removing and

mapping for a flat back $18 \%$ thick airfoil

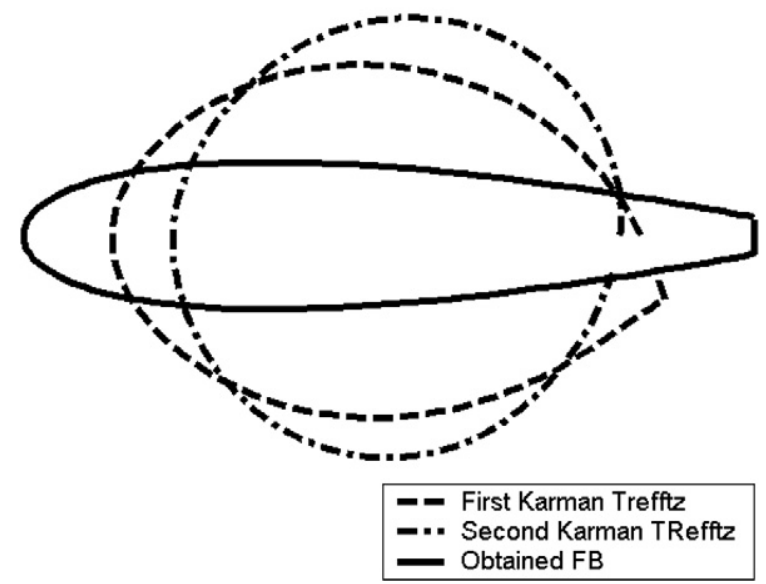

Fig. 11. Mapping of a Flat Back airfoil. Only 64 coefficients are used in the final mapping. The first and second Karman Trefftz transform are shown as described in Section 4. The use of an extra Karman Trefftz greatly simplifies the quasi circle mapping.

\section{Comparison between the KarmanTrefftz and Laurent's series expansion for a Gurney flap}

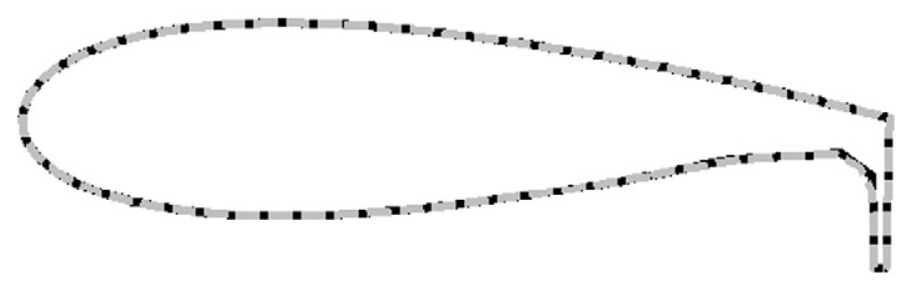

\section{- Mapping with Karman Treffz \\ Laurent's series}

Fig. 12. Comparison between pure mapping (Eqs. (51)-(55)) and Laurent's series decomposition (Eq. (56)) for a non-smooth Gurney flap airfoil. In this extreme case 265 coefficients are used.

\subsection{Efficiency of the corner remover procedure}

Three examples of profiles that can be handled by application of the iterative Karman Trefftz procedure are shown in Figs. 11-13. A comparison with the Laurent's series development is also given. As can be seen in Figs. 12 and 13, the accuracy decreases even with 258 coefficients in the case of the Gurney flap and the spoiler case. In such cases it may be worthwhile to retain the last Karman Trefftz transformations to limit the number of coefficients. For the case of the flat back airfoil, see Fig. 11, the results were still accurate using only 64 coefficients.

\subsection{Multicomponent mapping}

The first implementation of the multicomponent mapping consists of a recovering of the Suddhoo-Hall [33] airfoil to compare with the known exact pressure distribution. Fig. 14 shows the pressure coefficient isolines around the full four element airfoil obtained by direct evaluation of Eq. (19). The inviscid flow calculation which will be given by the many circles flow, obtained using 64 coefficients and evaluated on 1000 points on each airfoil. The resulting accuracy is $10^{-11}$ in terms of fulfillment of the boundary condition on all airfoil surfaces. The pressure coefficients, see Fig. 15, are in very good agreement with the known analytical solution [33]. Fig. 16 shows how the solution for one point 


\section{Comparison between the Karman Trefftz and the Laurent's series expansion for an airfoil with spoiler}

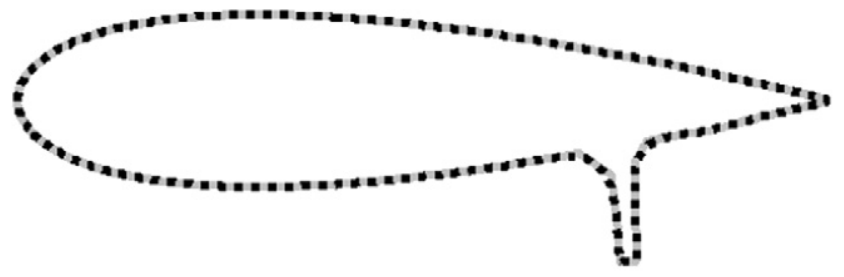

Mapping with Karman Trefftz .... Laurent's series

Fig. 13. Comparison between pure mapping (Eqs. (51)-(55)) and Laurent's series decomposition (Eq. (56)) for a non-smooth airfoil with open spoiler. 265 coefficients are also used.

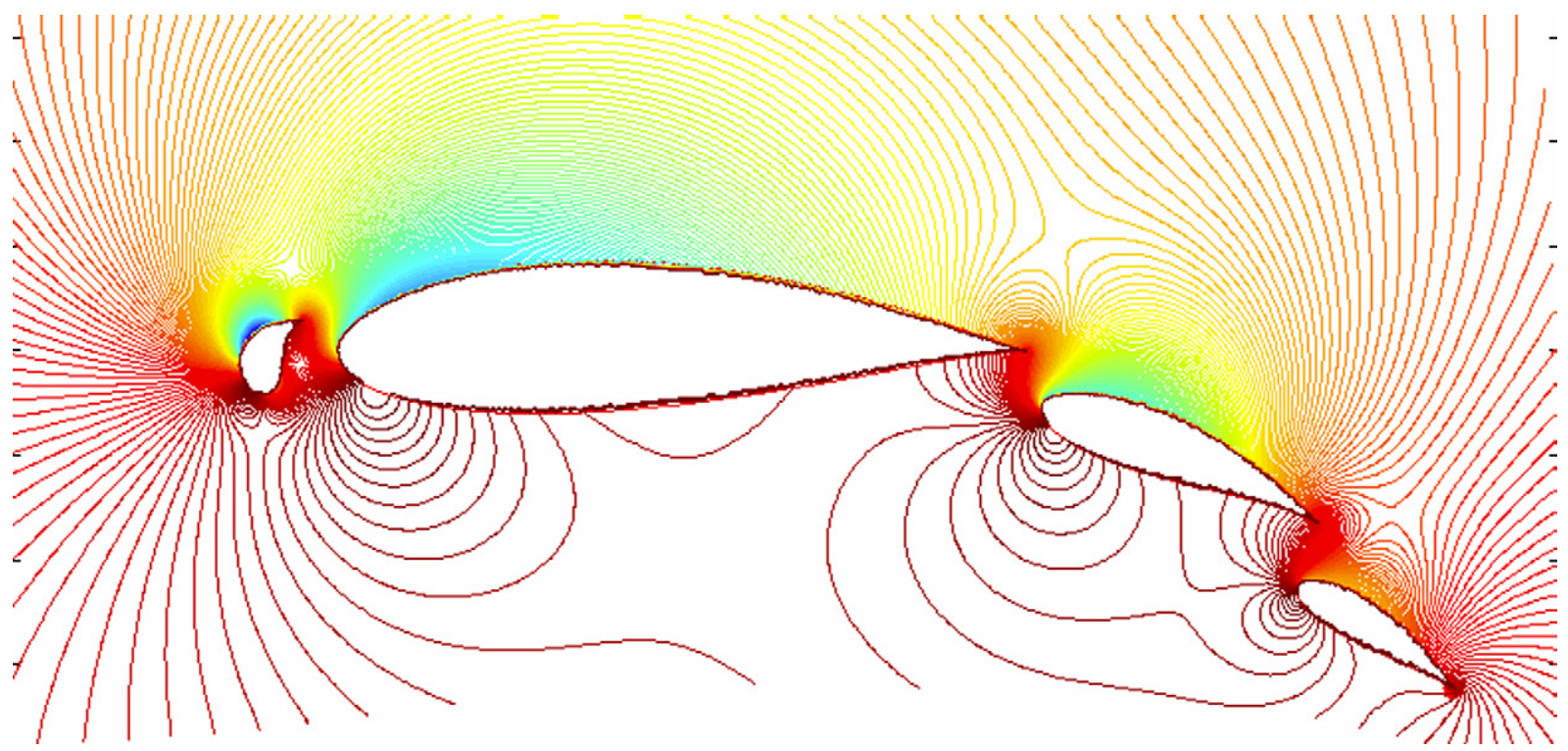

Fig. 14. Normalized pressure coefficients for the test case Suddhoo-Hall airfoil. The isolines are obtained by a polar grid before the mapping. 64 coefficients are used to represent each airfoil in Eq. (7).

vortex placed in a uniform flow around the four circles of Fig. 7 is transferred to the more complicated problem of a point vortex close to the Suddhoo-Hall airfoil.

A typical two element section front wing of an F3 race car in ground effect has been studied [34]. The ground is represented by an image of the two element section. The resulting inviscid pressure distribution is shown in Fig. 17. These results agree very well with other multielement linear panel codes. Fig. 18 shows the streamlines deduced from the circles in Fig. 6 by application of the conformal mapping transform. It shows the ability of the procedure to treat directly and efficiently complicated flow problems by exploiting the general, standard form of the circle solution.

\section{Conclusion}

A powerful parameterized method to map any shape of multicomponent geometry in two dimensions has been developed. The construction of the mapping demands low computational time. The model has been benchmarked 


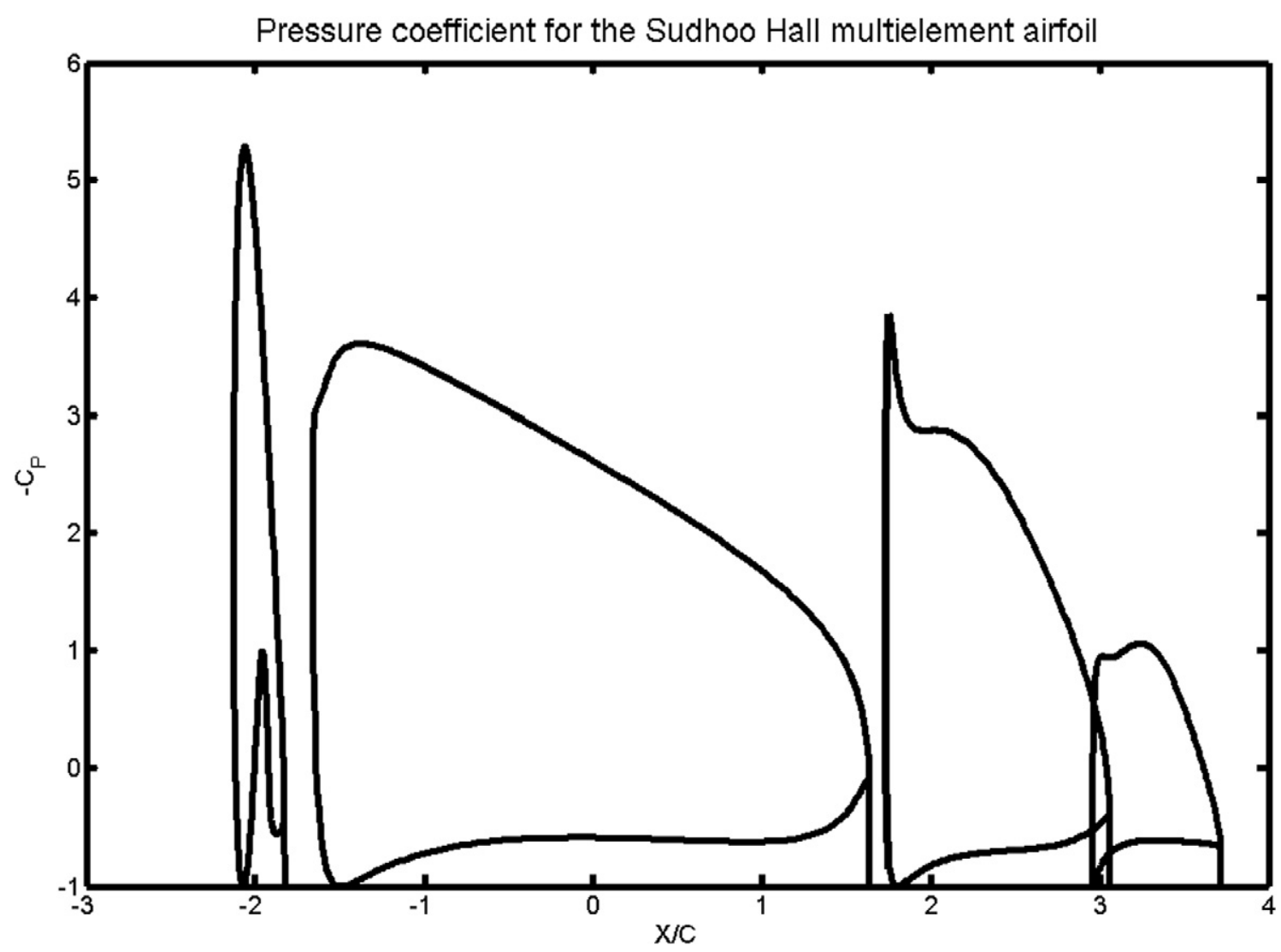

Fig. 15. Pressure coefficient on each of the four wings. Contrary to the panel method, even if the pressure is evaluated in 1000 points on each wing, no unphysical harmonic appear even if 64 coefficients only are used. The pressure is in excellent agreement with the exact solution.

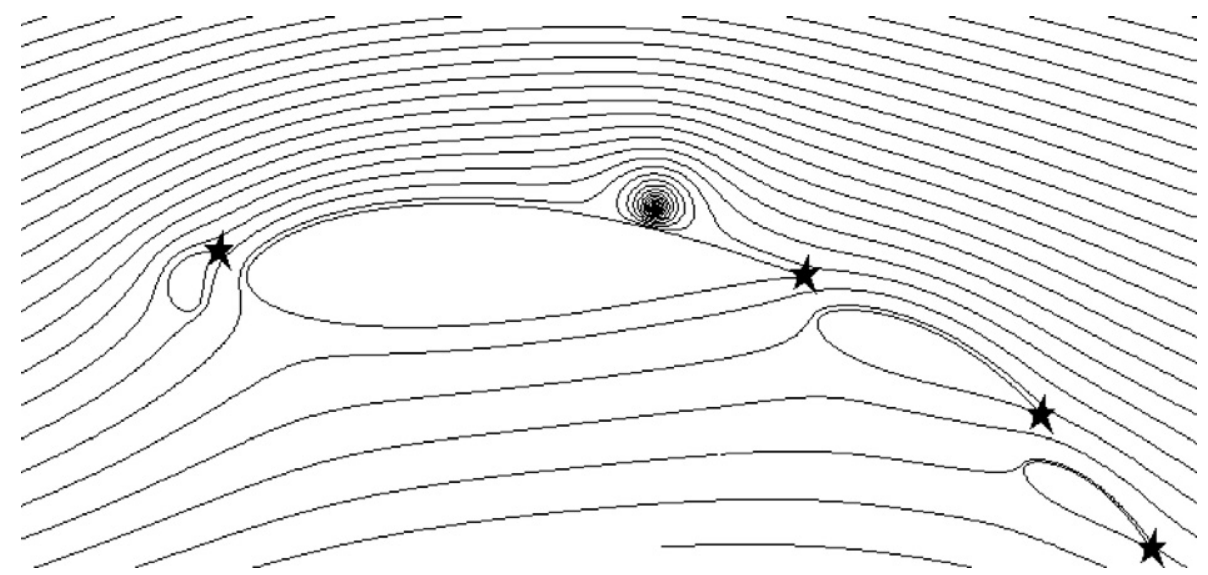

Fig. 16. Suddhoo-Hall airfoil with a logarithmic singularity close to the main element. This figure has been directly derived after a mapping of Fig. 7.

against analytical solutions and several other numerical solutions by other schemes. The full procedure has been shown to be preferable for vortex flow simulations over the standard panel methods. The field of possible application of the mapping is vast, ranging from steady inviscid aerodynamics to unsteady vortex flows as well as to other areas of physics. This mapping, with its abilities in terms of airfoil shaping, may provide significant advances in the studies 


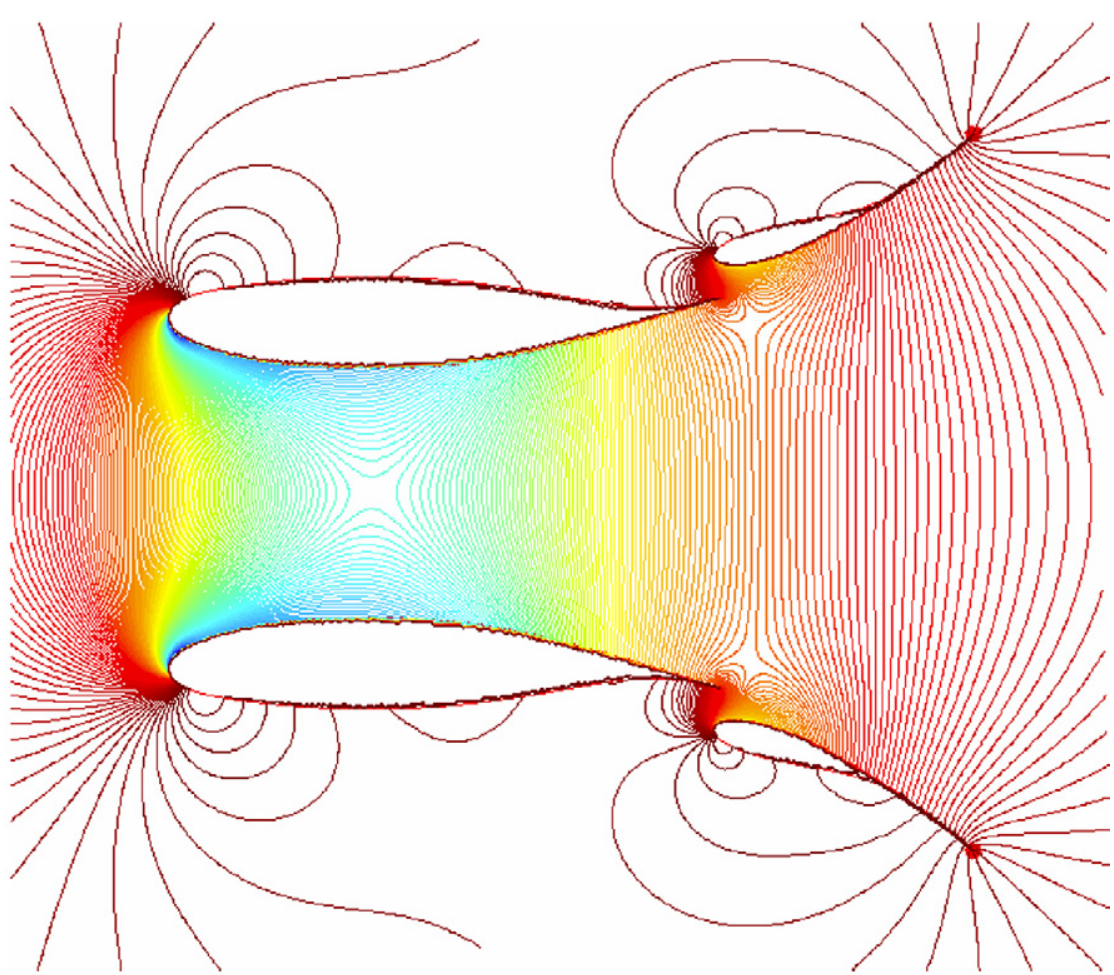

Fig. 17. Normalized pressure distribution for an F3 wing in ground effect.

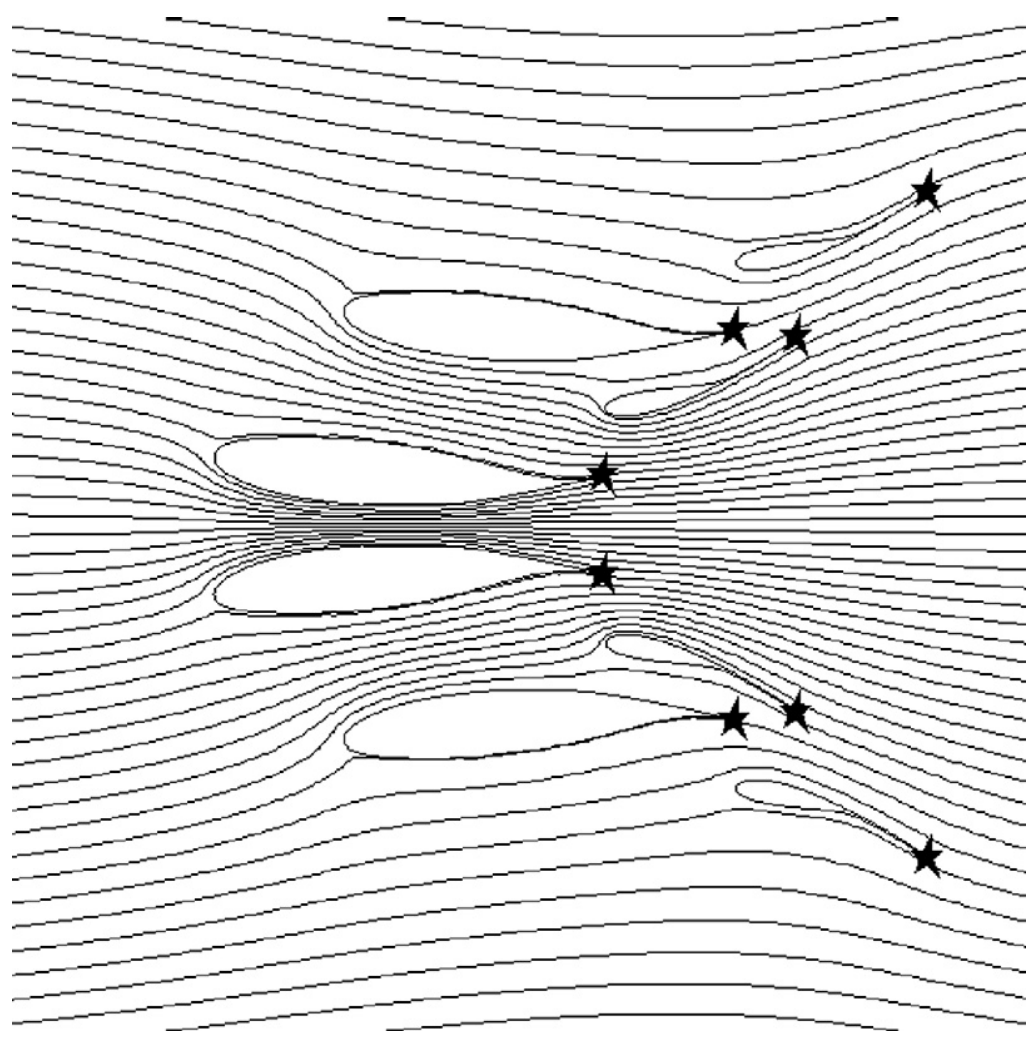

Fig. 18. From Fig. 6 the eight circles are mapped onto two F3 systems in ground effect. 
of complicated vortex flows in complicated geometries. In addition, this conformal map can model aero elasticity and morphing wing in a smooth manner.

\section{Acknowledgements}

Stefan Engblom from the Department of Information Technology at Uppsala University is deeply acknowledged for the development of the FMM method used in the present paper. The authors are grateful to Prof Hassan Aref and Dr. Jens Honore Walther from the Technical University of Denmark for fruitful discussions.

The Swedish Energy Agency (STEM) and the Swedish agency for innovations systems (VINNOVA) have partly financed this study.

\section{Appendix A. NACA0018 parameters}

$$
\begin{aligned}
& b=0.288063378302759 \\
& \sigma=-0.0239725168283033 \\
& C \text { coefficients } \\
& 0.207510672717152 \\
& 0.0214921733128163 \\
& 0.0030410530485142 \\
& 0.00132688739096312 \\
& 0.000710107218975926 \\
& 0.000504919658701561 \\
& 0.000247504662316063 \\
& 0.000207118282407516 \\
& 0.000118355764179746 \\
& 0.000103707003865087 \\
& 6.6811057626876 \mathrm{e}-005 \\
& 5.77364471616596 \mathrm{e}-005 \\
& 4.2129489106887 \mathrm{e}-005 \\
& 3.48926336578873 \mathrm{e}-005 \\
& 2.77959793437947 \mathrm{e}-005 \\
& 2.23787031673679 \mathrm{e}-005 \\
& 1.99116110901332 \mathrm{e}-005 \\
& 1.4934467651731 \mathrm{e}-005 \\
& 1.39070061787088 \mathrm{e}-005 \\
& 1.05226606205922 \mathrm{e}-005 \\
& 1.04818943700456 \mathrm{e}-005 \\
& 7.32045450815833 \mathrm{e}-006 \\
& 7.49673215499734 \mathrm{e}-006 \\
& 5.75711826933165 \mathrm{e}-006 \\
& 5.73162649729149 \mathrm{e}-006 \\
& 3.99924486662546 \mathrm{e}-006 \\
& 4.19863819870184 \mathrm{e}-006 \\
& 3.55526553622761 \mathrm{e}-006 \\
& 3.23883720133558 \mathrm{e}-006 \\
& 2.55155302477992 \mathrm{e}-006 \\
& 2.56957921985826 \mathrm{e}-006 \\
& 2.30800285349393 \mathrm{e}-006 \\
& 1.68508455540682 \mathrm{e}-006 \\
& 1.51081287350312 \mathrm{e}-006 \\
& 1.49369384329867 \mathrm{e}-006 \\
& 1.43656326567736 \mathrm{e}-006
\end{aligned}
$$


$9.92984669724092 \mathrm{e}-007$

$1.11120663158771 \mathrm{e}-006$

$1.06524575495901 \mathrm{e}-006$

$8.71180376642054 \mathrm{e}-007$

$5.42948312161243 \mathrm{e}-007$

$6.7001373078819 \mathrm{e}-007$

$6.41367639836314 \mathrm{e}-007$

$4.57297481080884 \mathrm{e}-007$

$3.77773912040894 \mathrm{e}-007$

$4.87404673833735 \mathrm{e}-007$

$4.23466648890017 \mathrm{e}-007$

$2.25808280933572 \mathrm{e}-007$

$2.38975057132785 \mathrm{e}-007$

$2.87251216701987 \mathrm{e}-007$

$2.18546207509777 \mathrm{e}-007$

$1.10596153435073 \mathrm{e}-007$

$1.71038548615424 \mathrm{e}-007$

$1.73227436098123 \mathrm{e}-007$

$9.76932695586293 \mathrm{e}-008$

$4.99882080923025 \mathrm{e}-008$

$8.89254212747578 \mathrm{e}-008$

$6.94326647709732 \mathrm{e}-008$

$2.71878885727139 \mathrm{e}-008$

$2.10989491861505 \mathrm{e}-008$

$2.88295914450696 \mathrm{e}-008$

$1.03428048259863 \mathrm{e}-008$

\section{References}

[1] S.G. Krantz, in: Handbook of Complex Variables, Birkhäuser, Boston, MA, 1999, pp. 86-87.

[2] D. Gaier, Konstruktive Methoden der konformen Abbildung, Springer, Berlin, 1964.

[3] F.L. Ponta, The kinematic Laplacian equation method, Journal of Computational Physics 207 (2) (2005) 405-426.

[4] T. Theodorsen, I.E. Garrick, General potential theory of arbitrary wing sections, N.A.C.A., T.R. No 452, 1934.

[5] D.C. Ives, A modern look at conformal mapping including multiply connected regions, AIAA Journal 14 (1976) 1006-1011.

[6] R. Eppler, Airfoil program system Profil00 user's guide, Richard Eppler c.2000.

[7] M.S. Selig, M.D. Maughmer, Generalized multipoint inverse airfoil design, AIAA Journal 30 (1992) 2618-2625.

[8] I. E. Garrick, On the plane potential flow past a lattice of arbitrary airfoils, N.A.C.A., T.R. No. 788, 1944.

[9] N.D. Halsey, Potential flow analysis of multielement airfoils using conformal mapping, AIAA J ournal (1979) $79-0271$.

[10] R. Wegmann, An iterative method for the conformal mapping of doubly connected regions, Journal of Computational and Applied Mathematics 14 (1986) 79-98.

[11] G. Morgenthal, Aerodynamic analysis of structures using high-resolution vortex particle methods, PhD thesis Magdalene college, University of Cambridge, 2002.

[12] T. Sarpkaya, An inviscid model of two dimensional vortex shedding for transient and asymptotically steady separated flow over and inclined flat plate, Journal of Fluid Mechanics 68 (March 1975) 109-128.

[13] C. Shih, L. Lourenco, L. Van Dommelen, A. Krothapalli, Unsteady flow past an airfoil pitching at a constant rate, AIAA Journal 30 (1992) $1153-1161$.

[14] A.J. Chorin, Numerical of slightly viscous flows, Journal of Fluid Mechanics 57 (1973) 785-796, MR 52:16280.

[15] S. Subramaniam, A new mesh-free vortex method, PhD thesis Florida state University, 1996.

[16] J. Carrier, L. Greengard, V. Rokhlin, A fats adaptative multipole algorithm for particle simulations, SIAM Journal of Scientific and Statistical Computing (July 1988).

[17] J.L. Hess, A.M.O. Smith, Calculation of potential flow about arbitrary bodies, Progress in Aerospace Sciences 8 (1966) 1-138.

[18] R.I. Lewis, Vortex Element Methods for Fluid Dynamics Analysis of Engineering Systems, Cambridge Engine Technology Series, vol. 1, Cambridge University Press, Cambridge, 1991.

[19] P. Ramachandran, Development and study of a high resolution two dimensional random vortex method, PhD thesis department of Aerospace engineering Indian institute of technology, Madras, 2004.

[20] L.M. Milne-Thomson, Theoretical Hydrodynamics, Macmillan Co., New York, 1967. 
[21] M.E. Klonowska, W.J. Prosnak, On an effective method for conformal mapping of multiply connected domains, Acta Mechanica 119 (1996) $35-52$.

[22] W.J. Prosnak, Computation of Fluid Motions in Multiply Connected Domains, Wissenschaft \& Tecnik, 1987.

[23] K. Streitlien, M.S. Triantafyllou, Force and moment on a Joukowsky profile in the presence of point vortices, AIAA Journal 33 (4) (April 1994).

[24] G. Couchet, Mouvement plan d'un fluide en présence d'un profil mobile, Memorial des Sciences Mathematiques Gauthier-Villars 135, Paris $135,1956$.

[25] D.G. Crowdy, Analytical solutions for uniform potential flow past multiple cylinders, European Journal of Mechanics B Fluids 25 (4) (2006) 459-470.

[26] D.G. Crowdy, Calculating the lift on a finite stack of cylindrical aerofoils, Proceedings of the Royal Society A 462 (January 2006) 1387-1407.

[27] B.R. Williams, An exact test case for the plane potential flow about two adjacent lifting airfoils, Aeronautical research council reports and memoranda No. 3717, 1971.

[28] R.R. Clements, An inviscid model of two dimensional vortex shedding, Journal of Fluid Mechanics 57 (2) (1973) $321-336$.

[29] P. Luchini, F. Manzo, Flow around simply and multiply connected bodies: a new iterative scheme for conformal mapping, AIAA Journal 27 (3) (1989) 345-351.

[30] S. Sarkar, K. Venkatraman, Numerical simulation of incompressible viscous flow past a heaving airfoil, International Journal for Numerical Methods in Fluids 51 (1) (2005) 1-29.

[31] J.H. Walther, private communication.

[32] M. Drela, XFOIL: An analysis and design system for low Reynolds number airfoils, in: Conference Proceedings on Low Reynolds Number Aerodynamics, Notre Dame, Indiana, 5-7 June 1989.

[33] A. Suddhoo, I.M. Hall, Test cases for the plane potential flow past multi-element aerofoils, Aeronautical Journal 89 (1985) $403-414$.

[34] E.L.N. Terry, Extension of the aerodynamic design program MSES for the simulation of Boundary layer suction, MSc thesis, Delft university of technology, 2004. 